\title{
The case $k=2$ of the Shuffle Conjecture*
}

\author{
Adriano Garsia, Angela Hicks and Andrew Stout
}

It was conjectured in [5] and proved by Mark Haiman in [13] that the Frobenius Characteristic of the $S_{n}$ Module of Diagonal Harmonics is none other than $\nabla e_{n}$. Here $\nabla$ is the symmetric function operator introduced in [1] with eigen-functions the modified Macdonald basis $\left\{\tilde{H}_{\mu}\right\}_{\mu}$. The Shuffle Conjecture [12] expresses the scalar product $\left\langle\nabla e_{n}, h_{\mu_{1}} h_{\mu_{2}} \cdots h_{\mu_{k}}\right\rangle$ as a weighted sum of Parking Functions on the $n \times n$ lattice square which are shuffles of $k$ increasing words. In [10] Jim Haglund succeeded in proving the $k=2$ case of this conjecture. In this paper we give a new and more direct proof of the combinatorial part of Haglund's argument and obtain a substantial reduction in the complexity of the symmetric function part.

\section{Introduction}

In this writing it is convenient to represent Parking Functions in the $n \times n$ lattice square by two line arrays

$$
P F=\left[\begin{array}{llll}
v_{1} & v_{2} & \cdots & v_{n} \\
u_{1} & u_{2} & \cdots & u_{n}
\end{array}\right]
$$

with $u_{1}, u_{2}, \ldots, u_{n}$ integers satisfying $u_{1}=0$ and $0 \leq u_{i} \leq u_{i-1}+1$ and $V=\left(v_{1}, v_{2}, \ldots, v_{n}\right)$ a permutation in $S_{n}$ satisfying

$$
u_{i}=u_{i-1}+1 \Longrightarrow v_{i}>v_{i-1} \text {. }
$$

Here and after, the collection of Parking Functions on $1,2, \ldots, n$ will be denoted $\mathcal{P} \mathcal{F}_{n}$. We will denote by $\sigma(P F)$ the permutation obtained by successive right to left readings of the components of the vector $V=\left(v_{1}, v_{2}, \ldots, v_{n}\right)$ according to decreasing values of $u_{1}, u_{2}, \ldots, u_{n}$. We will here and after refer to $\sigma(P F)$ as the "diagonal permutation" of $P F$. It will also be convenient

\footnotetext{
${ }^{*}$ Work carried out under NSF support.
} 
to let $i \operatorname{des}(P F)$ denote the descent set of the inverse of $\sigma(P F)$. We also set

$$
\operatorname{area}(P F)=\sum_{i=1}^{n} u_{i} q \text { and }
$$

$$
\operatorname{dinv}(P F)=\sum_{1 \leq i<j \leq n}\left(\chi\left(u_{i}=u_{j} \& v_{i}<v_{j}\right)+\chi\left(u_{i}=u_{j}+1 \& v_{i}>v_{j}\right)\right)
$$

This given, each Parking Function is assigned the "weight"

$$
w(P F)=t^{\operatorname{area}(P F)} q^{\operatorname{dinv}(P F)} Q_{i d e s(P F)}[X]
$$

where for $S \subseteq\{1,2, \ldots, n-1\}, Q_{S}[X]$ denotes the corresponding Gessel [9] fundamental quasi-symmetric function in $x_{1}, x_{2}, \ldots, x_{n}$.

Parking functions are endowed by a colorful history and a jargon (see for instance [11]) that is very helpful in dealing with them combinatorially as well as analytically. For us it is sufficient to be able to translate properties of the two line array to visual properties of the corresponding tableau. A single example of this correspondence should be sufficient for our purposes.

In the figure below we have a Parking Function as it is usually depicted together with the corresponding vector $U=\left(u_{1}, u_{2}, \ldots, u_{n}\right)$ on its left and its corresponding vector $V=\left(v_{1}, v_{2}, \ldots, v_{n}\right)$ on its right.

$$
P F=\left[\begin{array}{llllllll}
4 & 6 & 8 & 1 & 3 & 2 & 7 & 5 \\
0 & 1 & 2 & 2 & 3 & 0 & 1 & 1
\end{array}\right] \Longleftrightarrow
$$

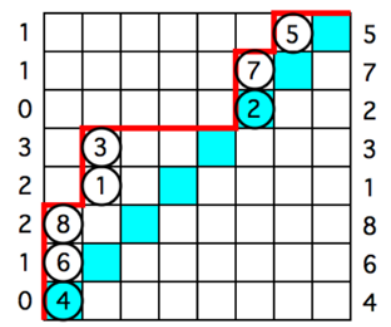

The diagonal of shaded cells is usually referred to as "main diagonal" (or 0-diagonal) of PF. The numbers in the lattice cells are the "cars". The path along whose vertical steps we have set the cars is the "Dyck" path "supporting" $P F$. This given, the components of $U=\left(u_{1}, u_{2}, \ldots, u_{n}\right)$ are none other than the orders of the diagonals containing the cars. In this case car 3 is in the third diagonal, 1 and 8 are in the second diagonal, 5, 7 and 6 are in the first diagonal and 2 and 4 are in the main diagonal. We have purposely listed the cars by diagonals from right to left starting with the highest diagonal. This gives the diagonal permutation $\sigma(P F)$. It is clear from this imagery, that the first sum in (1.1) gives the total number of cells 
between the supporting Dyck path and the main diagonal. We also see that two cars in the same diagonal with the car on the left smaller than the car on the right will contribute a unit to $\operatorname{dinv}(P F)$. The same holds true when a car on the left is bigger than a car on the right with the latter in the adjacent lower diagonal. It will be convenient to think that the parking functions, with a given Dyck path $D$ in the $n \times n$ lattice square, are constructed by first placing circles along the of NORTH steps of $D$ and then filling the circles with $1,2, \ldots, n$ in a column increasing manner.

The so called "Shuffle Conjecture" asserts that

$$
\nabla e_{n}=\sum_{P F \in \mathcal{P} \mathcal{F}_{n}} t^{\operatorname{area}(P F)} q^{\operatorname{dinv}(P F)} Q_{i d e s(P F)}[X] .
$$

Here $\nabla$ is the symmetric function operator defined in [1] by setting for the modified Macdonald basis $\left\{\tilde{H}_{\mu}[X ; q, t]\right\}_{\mu}$ introduced in [4]

$$
\nabla \tilde{H}_{\mu}[X ; q, t]=t^{n(\mu)} q^{n\left(\mu^{\prime}\right)} \tilde{H}_{\mu}[X ; q, t] \quad\left(n(\mu)=\sum_{i=1}^{k}(i-1) \mu_{i}\right) .
$$

From a result of Gessel [9] it follows that (1.2) is equivalent to the following identity holding true for all partitions $\mu=\left(\mu_{1}, \mu_{2}, \ldots, \mu_{k}\right)$

$$
\begin{aligned}
& \left\langle\nabla e_{n}, h_{\mu_{1}} h_{\mu_{2}} \cdots h_{\mu_{k}}\right\rangle \\
& \quad=\sum_{P F \in \mathcal{P} \mathcal{F}_{n}} t^{\operatorname{area}(P F)} q^{\operatorname{dinv}(P F)} \chi\left(\sigma(P F) \in E_{1} w E_{2} w \cdots \omega E_{k}\right)
\end{aligned}
$$

where $h_{\mu_{1}} h_{\mu_{2}} \cdots h_{\mu_{k}}$ is the "homogeneous" symmetric function basis indexed by $\mu, E_{1}, E_{2}, \ldots, E_{k}$ are successive segments of the word $1234 \cdots n$ of respective lengths $\mu_{1}, \mu_{2}, \ldots, \mu_{k}$ and " $\chi\left(\sigma(P F) \in E_{1} w E_{2} w \cdots \omega E_{k}\right)$ " is to indicate that the sum is to be carried out over parking functions in $\mathcal{P} \mathcal{F}_{n}$ whose diagonal word is a shuffle of the words $E_{1}, E_{2}, \ldots, E_{k}$.

The $k=2$ case of the Shuffle conjecture may be stated as follows

Theorem 1.1 (J. Haglund). For all $0 \leq j<n$ we have

$$
\begin{aligned}
& \left\langle\nabla e_{n}, h_{j} h_{n-j}\right\rangle \\
& \quad=\sum_{P F \in \mathcal{P F}_{n}} t^{\operatorname{area}(P F)} q^{\operatorname{dinv}(P F)} \chi(\sigma(P F) \in 12 \cdots j \cup \cup j+1 \cdots n)
\end{aligned}
$$

where the sum is over Parking Functions with diagonal permutation a shuffle of $12 \cdots j$ with $j+1 \cdots n$. 
In [10] this result is obtained as a corollary of the following sharper identity.

Theorem 1.2 (J. Haglund). For all $0 \leq j<n$ and $1 \leq k \leq n$ we have

$$
\begin{aligned}
& \left\langle\Delta_{h_{j}} E_{n k}, e_{n}\right\rangle \\
& \quad=\sum_{P F \in \mathcal{P F}_{n}(k)} t^{\operatorname{area}(P F)} q^{\operatorname{dinv}(P F)} \chi(\sigma(P F) \in 12 \cdots j \cup \cup j+1 \cdots j+n)
\end{aligned}
$$

where $\mathcal{P F}_{n}(k)$ is the collection of Parking Functions in the $n \times n$ lattice square that have $k$ of the cars $j+1, \ldots, j+n$ in the main diagonal with car $j+n$ in the cell $(1,1)$.

Here the $E_{n k}$ are certain remarkable symmetric functions introduced in [3] with sum

$$
E_{n 1}+E_{n 2}+\cdots+E_{n k}=e_{n}
$$

and $\Delta_{h_{j}}$ is the particular case $F=h_{j}$ of the operator obtained by setting (for a given symmetric function $F$ )

$$
\Delta_{F} \tilde{H}_{\mu}[X ; q, t]=F\left[\sum_{(i, j) \in \mu} t^{i-1} q^{j-1}\right] \tilde{H}_{\mu}[X ; q, t] .
$$

The identity in (1.4) is proved by showing first that the polynomials

$$
H_{j, n, k}=\left\langle\Delta_{h_{j}} E_{n k}, e_{n}\right\rangle
$$

satisfy the recursion

$$
H_{j, n, k}=t^{n-k} \sum_{s=1}^{j}\left[\begin{array}{c}
k+s-1 \\
s
\end{array}\right]_{q} H_{n-k, j, s}
$$

and the proof is then completed by showing that the right-hand side of (1.5) satisfies the same recursion and the same initial conditions.

Exploring possible extensions of the Haglund result we were led to study the original argument in the minutest detail. This turned to be a remarkably forbidding task for the final results is obtained in [10] via a tortuous path through a forest of highly non-trivial symmetric function identities. This prompted us to find ways to simplify the original argument by shortening the steps and making them more transparent whenever possible.

This effort ultimately paid off even more than we ever expected. The tangible by-products are firstly a shorter derivation of Theorem 1.1 from 
Theorem 1.2 and a shorter, simpler and more transparent proof of Theorem 1.2, including a direct bijective proof that the combinatorial side of (1.5) satisfies the recursion in (1.6). Secondly, the discovery by computer data of the possibility of a substantial refinement of the identity in (1.4) which we plan to pursue in our future investigations.

Much as we would like to have our presentation self contained, we will need to use, without proof a number of Macdonald polynomial identities established in [2, 6], and [7]. For the benefit of researchers that may wish to work in this fascinating area of Algebraic Combinatorics we present in section 1 a collection of identities in Macdonald Polynomial Theory which we use here and have been proved useful in past work.

In the second section we derive the additional identities we need to prove (1.3) and (1.4). In the third section we prove (1.4) which is the symmetric function side of the recursion and determine the corresponding initial conditions. In the fourth and final section we prove the right-hand side (1.5) satisfies the same recursion by means of our direct bijection and thereby complete the proof of Theorems 1.1 and 1.2.

Our end product should be a considerably less painful derivation of (1.4) than the one given in [10].

\section{A Macdonald Polynomial tool kit}

The space of symmetric polynomials will be denoted $\Lambda$. The subspace of homogeneous symmetric polynomials of degree $m$ will be denoted $\Lambda^{=m}$. It will also be convenient to let $\Lambda^{\leq m}$ denote the subspace of symmetric polynomials that are of degree $\leq m$. We will seldom work with symmetric polynomials expressed in terms of variables but rather express them in terms of one of the six classical symmetric function bases: "power" $\left\{p_{\mu}\right\}_{\mu}$, "monomial" $\left\{m_{\mu}\right\}_{\mu}$, "homogeneous" $\left\{h_{\mu}\right\}_{\mu}$, "elementary" $\left\{e_{\mu}\right\}_{\mu}$, "forgotten" $\left\{f_{\mu}\right\}_{\mu}$, and "Schur" $\left\{s_{\mu}\right\}_{\mu}$.

We recall that the fundamental involution $\omega$ may be defined by setting for the power basis indexed by $\mu=\left(\mu_{1}, \mu_{2}, \ldots, \mu_{k}\right) \vdash n$

$$
\omega p_{\mu}=(-1)^{n-k} p_{\mu}=(-1)^{|\mu|-l(\mu)} p_{\mu}
$$

where for any vector $v=\left(v_{1}, v_{2}, \ldots, v_{k}\right)$ we set $|v|=\sum_{i=1}^{k} v_{i}$ and $l(v)=k$.

In dealing with symmetric function identities, specially with those arising in the Theory of Macdonald Polynomials, we find it convenient and often indispensable to use plethystic notation. This device has a straightforward 
definition which can be verbatim implemented in MAPLE or MATHEMATICA. We simply set for any expression $E=E\left(t_{1}, t_{2}, \ldots\right)$ and any power symmetric function $p_{k}$

$$
p_{k}[E]=E\left(t_{1}^{k}, t_{2}^{k}, \ldots\right)
$$

This given, for any symmetric function $F$ we set

$$
F[E]=\left.Q_{F}\left(p_{1}, p_{2}, \ldots\right)\right|_{p_{k} \rightarrow E\left(t_{1}^{k}, t_{2}^{k}, \ldots\right)}
$$

where $Q_{F}$ is the polynomial yielding the expansion of $F$ in terms of the power basis. Note that in writing $E\left(t_{1}, t_{2}, \ldots\right)$ we are tacitly assuming that $t_{1}, t_{2}, t_{3}, \ldots$ are all the variables appearing in $E$ and in writing $E\left(t_{1}^{k}, t_{2}^{k}, \ldots\right)$ we intend that all the variables appearing in $E$ have been raised to their $k^{\text {th }}$ power.

A paradoxical but necessary property of plethystic substitutions is that (2.1) requires

$$
p_{k}[-E]=-p_{k}[E]
$$

This notwithstanding, we will still need to carry out ordinary changes of signs. To distinguish it from the "plethystic" minus sign, we will carry out the "ordinary" sign change by prepending our expressions with a superscripted minus sign, or as the case may be, by means of a new variables $\epsilon$ which outside of the plethystic bracket is simply replaced by -1 . For instance, these conventions give for $X_{k}=x_{1}+x_{2}+\cdots+x_{n}$

$$
p_{k}\left[-^{-} X_{n}\right]=(-1)^{k-1} \sum_{i=1}^{n} x_{i}^{k}
$$

or, equivalently

$$
p_{k}\left[-\epsilon X_{n}\right]=-\epsilon^{k} \sum_{i=1}^{n} x_{i}^{k}=(-1)^{k-1} \sum_{i=1}^{n} x_{i}^{k} .
$$

In particular we get for $X=x_{1}+x_{2}+x_{3}+\cdots$

$$
\omega p_{k}[X]=p_{k}\left[{ }^{-} X\right]
$$

Thus for any symmetric function $F \in \Lambda$ and any expression $E$ we have

$$
\omega F[E]=F\left[-^{-} E\right]=F[-\epsilon E] .
$$


In particular, if $F \in \Lambda^{=k}$ we may also rewrite this as

$$
\left.F[-E]=\omega F^{-} E\right]=(-1)^{k} \omega F[E] .
$$

The formal power series

$$
\Omega=\exp \left(\sum_{k \geq 1} \frac{p_{k}}{k}\right)
$$

combined with plethysic substitutions will provide a powerful way of dealing with the many generating functions occurring in our manipulations.

For a given expression $E$ we will set

$$
\Omega[E]=\exp \left(\sum_{k \geq 1} \frac{p_{k}[E]}{k}\right)
$$

and since for any two expressions $A, B(2.1)$ gives

$$
p_{k}[A+B]=p_{k}[A]+p_{k}[B] .
$$

We derive from this the fundamental formula

$$
\Omega[A+B]=\Omega[A] \Omega[B] .
$$

In particular when $A=\sum_{i=1}^{n} a_{i}$ and $B=\sum_{j=1}^{m} b_{j}$ we get

$$
\Omega[t A-t B]=\frac{\prod_{j=1}^{m}\left(1-t b_{j}\right)}{\prod_{i=1}^{n}\left(1-t a_{i}\right)} .
$$

Clearly, for any two expressions $A, B$ we can also view $\Omega[t(A-B)]$ as the generating functions of the homogeneous symmetric functions plethystically evaluated at $A-B$

$$
\Omega[t(A-B)]=\sum_{m \geq 1} t^{m} h_{m}[A-B] .
$$

In particular, by equating coefficients of $t^{m}$ on both sides of (2.9), (2.7) gives (using (2.6))

$$
h_{m}[A-B]=\sum_{r=0}^{m} h_{m-r}[A] h_{r}[-B]=\sum_{r=0}^{m} h_{m-r}[A](-1)^{r} e_{r}[B]
$$

which is an identity that will play a crucial role in many of our calculations. 


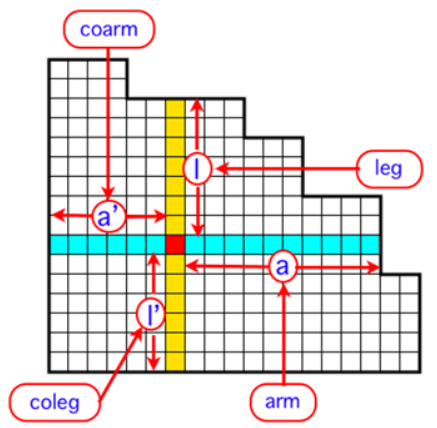

Figure 2.1

To present our Macdonald polynomial kit, it is convenient to identify partitions with their (french) Ferrers diagram. Given a partition $\mu$ and a cell $c \in \mu$, Macdonald introduces four parameters $l=l_{\mu}(c), l^{\prime}=l_{\mu}^{\prime}(c)$, $a=a_{\mu}(c)$ and $a^{\prime}=a_{\mu}^{\prime}(c)$ called leg, coleg, arm and coarm which give the number of lattice cells of $\mu$ strictly NORTH, SOUTH, EAST, and WEST of $c$, (see Figure 2.1).

Following Macdonald we will set

$$
n(\mu)=\sum_{c \in \mu} l_{\mu}(c)=\sum_{c \in \mu} l_{\mu}^{\prime}(c)=\sum_{i=1}^{l(\mu)}(i-1) \mu_{i} .
$$

Denoting by $\mu^{\prime}$ the conjugate of $\mu$, the basic ingredients playing a role in the theory of Macdonald polynomials are

$$
\begin{gathered}
T_{\mu}=t^{n(\mu)} q^{n\left(\mu^{\prime}\right)}, \quad B_{\mu}(q, t)=\sum_{c \in \mu} t^{l_{\mu}^{\prime}(c)} q^{a_{\mu}^{\prime}(c)}, \\
\Pi_{\mu}(q, t)=\prod_{c \in \mu ; c \neq(0,0)}\left(1-t^{l_{\mu}^{\prime}(c)} q^{a_{\mu}^{\prime}(c)}\right), \quad \text { and } \\
w_{\mu}(q, t)=\prod_{c \in \mu}\left(q^{a_{\mu}(c)}-t^{l_{\mu}(c)+1}\right)\left(t^{l_{\mu}(c)}-q^{a_{\mu}(c)+1}\right),
\end{gathered}
$$

together with a deformation of the Hall scalar product, which we call the "star" scalar product, defined by setting for the power basis

$$
\left\langle p_{\lambda}, p_{\mu}\right\rangle_{*}=(-1)^{|\mu|-l(\mu)} \prod_{i}\left(1-t^{\mu_{i}}\right)\left(1-q^{\mu_{i}}\right) z_{\mu} \chi(\lambda=\mu),
$$

where $z_{\mu}$ gives the order of the stabilizer of a permutation with cycle structure $\mu$. 
This given, the modified Macdonald Polynomials we will deal with here are the unique symmetric function basis $\left\{\tilde{H}_{\mu}(X ; q, t)\right\}_{\mu}$ which upper triangularly related to the modified Schur basis $\left\{s_{\lambda}[X /(t-1)]\right\}$ and satisfies the orthogonality condition

$$
\left\langle\tilde{H}_{\lambda}, \tilde{H}_{\mu}\right\rangle_{*}=\chi(\lambda=\mu) w_{\mu}(q, t) .
$$

The $*$-scalar product, is simply related to the ordinary Hall scalar product by setting for all pairs of symmetric functions $f, g$

$$
\langle f, g\rangle_{*}=\langle f, \omega \phi g\rangle
$$

where it has been customary to let $\phi$ be the operator defined by setting for any symmetric function $f$

$$
\phi f[X]=f[M X]
$$

with

$$
M=(1-t)(1-q) .
$$

Note that the inverse of $\phi$ is usually written in the form

$$
f^{*}[X]=f[X / M]
$$

In particular we also have for all symmetric functions $f, g$

$$
\langle f, g\rangle=\left\langle f, \omega g^{*}\right\rangle_{*} .
$$

The orthogonality relations in (2.12) yield the "Cauchy" identity for our Macdonald polynomials in the form

$$
\Omega\left[-\epsilon \frac{X Y}{M}\right]=\sum_{\mu} \frac{\tilde{H}_{\mu}[X] \tilde{H}_{\mu}[Y]}{w_{\mu}}
$$

which restricted to its homogeneous component of degree $n$ in $X$ and $Y$ reduces to

$$
e_{n}\left[\frac{X Y}{M}\right]=\sum_{\mu \vdash n} \frac{\tilde{H}_{\mu}[X] \tilde{H}_{\mu}[Y]}{w_{\mu}} .
$$


In fact, from the definition in (2.11) it follows that the reproducing kernel for the $*$-scalar product is given the sum

$$
\sum_{\mu}(-1)^{|\mu|-l(\mu)} \frac{p_{\mu}[X] p_{\mu}[Y]}{p_{\mu}[M]}=\sum_{\mu}(-1)^{|\mu|-l(\mu)} p_{\mu}\left[\frac{X Y}{M}\right]=\Omega\left[-\epsilon \frac{X Y}{M}\right]
$$

since the left-hand side of this identity must be equal to the right-hand side of (2.18) the equality in (2.18) must hold true as well. It will be convenient here and in the sequel to use the shorthand notation

$$
\widetilde{\Omega}\left[\frac{X Y}{M}\right]=\Omega\left[-\epsilon \frac{X Y}{M}\right] .
$$

A crucial tool which provides many of the transformations we will need in the sequel is the so-called Macdonald-Koorwinder "reciprocity" formula (see $[6,15]$ ). For our version of the Macdonald polynomials this formula can be written in the following concise form

$$
\frac{\tilde{H}_{\alpha}\left[1+u D_{\beta}\right]}{\prod_{c \in \alpha}\left(1-u t^{\prime} q^{a^{\prime}}\right)}=\frac{\tilde{H}_{\beta}\left[1+u D_{\alpha}\right]}{\prod_{c \in \beta}\left(1-u t^{l^{\prime}} q^{a^{\prime}}\right)} \quad(\text { for all pairs } \alpha, \beta)
$$

where for convenience we have set

$$
D_{\alpha}(q, t)=M B_{\alpha}(q, t)-1 \text {. }
$$

We will use here several special evaluations of (2.20). To begin, canceling the common factor $(1-u)$ out of the denominators on both sides of $(2.20)$ then setting $u=1$ gives

$$
\frac{\tilde{H}_{\alpha}\left[M B_{\beta}\right]}{\Pi_{\alpha}}=\frac{\tilde{H}_{\beta}\left[M B_{\alpha}\right]}{\Pi_{\beta}} \quad(\text { for all pairs } \alpha, \beta) .
$$

On the other hand replacing $u$ by $1 / u$ and letting $u=0$ in (2.20) gives

$$
\left.(-1)^{|\alpha|} \frac{\tilde{H}_{\alpha}\left[D_{\beta}\right]}{T_{\alpha}}=(-1)^{|\beta|} \frac{\tilde{H}_{\beta}\left[D_{\alpha}\right]}{T_{\beta}} \quad \text { (for all pairs } \alpha, \beta\right) .
$$

Since for $\beta$ the empty partition we can take $\tilde{H}_{\beta}=1$ and $D_{\beta}=-1,(2.20)$ in this case reduces to

$$
\tilde{H}_{\alpha}[1-u]=\prod_{c \in \alpha}\left(1-u t^{l^{\prime}} q^{a^{\prime}}\right)=(1-u) \sum_{r=0}^{n-1}(-u)^{r} e_{r}\left[B_{\mu}-1\right] .
$$


This identity yields the coefficients of hook Schur functions in the expansion.

$$
\tilde{H}_{\mu}[X ; q, t]=\sum_{\lambda \vdash|\mu|} s_{\mu}[X] \tilde{K}_{\lambda \mu}(q, t)=\sum_{\lambda \vdash|\mu|} s_{\mu}[X]\left\langle\tilde{H}_{\mu}, s_{\mu}\right\rangle .
$$

To see this we need the following auxiliary identity.

Lemma 2.1.

$$
s_{\mu}[1-u]= \begin{cases}(-u)^{r}(1-u) & \text { if } \mu=\left(n-r, 1^{r}\right), \\ 0 & \text { otherwise. }\end{cases}
$$

Proof. An application of (2.10) with $A=X$ and $B=u X$ gives

$$
\begin{aligned}
h_{m}[X-u X] & =\sum_{r=0}^{m} h_{m-r}[X] h_{r}[-u X] \\
& =\sum_{r=0}^{m} h_{m-r}[X] e_{r}[X](-u)^{r} \\
& =h_{m}[X]+\sum_{r=1}^{m}\left(s_{m-r, 1^{r}}[X]+s_{m-r+1,1^{r-1}}\right)(-u)^{r} \\
& =h_{m}[X]+\sum_{r=1}^{m} s_{m-r, 1^{r}}[X](-u)^{r}+\sum_{r=0}^{m-1} s_{m-r, 1^{r}}(-u)^{r+1} \\
& =h_{m}[X](1-u)+\sum_{r=1}^{m} s_{m-r, 1^{r}}[X](-u)^{r}(1-u) \\
& =\sum_{r=0}^{m} s_{m-r, 1^{r}}[X](-u)^{r}(1-u) .
\end{aligned}
$$

On the other hand the Cauchy formula gives

$$
h_{m}[X(1-u)]=\sum_{\mu \vdash m} s_{\mu}[X] s_{\mu}[1-u]
$$

and (2.26) follows by equating coefficients of $s_{\mu}[X]$.

Thus (2.25), with $X=1-u$, combined with (2.24) gives

$$
(1-u) \sum_{r=0}^{n-1}(-u)^{r} e_{r}\left[B_{\mu}-1\right]=\sum_{\lambda \vdash|\mu|} s_{\mu}[1-u]\left\langle\tilde{H}_{\mu}, s_{\mu}\right\rangle
$$


and (2.26) yields

$$
\left\langle\tilde{H}_{\mu}, s_{\left(n-r, 1^{r}\right)}\right\rangle=e_{r}\left[B_{\mu}-1\right]
$$

Finally, from the identity $e_{r} h_{n-r}=s_{\left(n-r, 1^{r}\right)}+s_{\left(n-r-1,1^{r-1}\right)}$ it follows that

$$
\left\langle\tilde{H}_{\mu}, e_{r} h_{n-r}\right\rangle=e_{r}\left[B_{\mu}\right]
$$

The case $\beta=(1)$ of $(2.22)$ is also significant in that it reduces to the identity

$$
\tilde{H}_{\alpha}[M]=M B_{\alpha} \Pi_{\alpha}
$$

Now it was conjectured in [5] and proved in [13] that the bigraded Frobenius characteristic of the diagonal Harmonics of $S_{n}$ is given by the symmetric function

$$
D H_{n}[X ; q, t]=\sum_{\mu \vdash n} \frac{T_{\mu} \tilde{H}_{\mu}(X ; q, t) B_{\mu}(q, t) \Pi_{\mu}(q, t)(1-t)(1-q)}{w_{\mu}(q, t)} .
$$

Surprisingly the intricate rational function on the right-hand side is none other than $\nabla e_{n}$. To see this we simply combine the relation in (2.30) with the degree $n$ restricted Cauchy formula (2.19), obtaining

$$
e_{n}[X]=e_{n}\left[\frac{X M}{M}\right]=\sum_{\mu \vdash n} \frac{\tilde{H}_{\mu}(X ; q, t) B_{\mu}(q, t) \Pi_{\mu}(q, t)(1-t)(1-q)}{w_{\mu}(q, t)}
$$

and (1.3) gives

$$
D H_{n}[X ; q, t]=\nabla e_{n}
$$

This discovery is precisely what led to the introduction of $\nabla$.

Our final ingredients we need, to carry out our proofs, are the coefficients $d_{\mu \nu}$ and $c_{\mu \nu}$ occurring in the Pieri formulas
a) $e_{1} \tilde{H}_{\nu}=\sum_{\mu \mu \nu} d_{\mu \nu} \tilde{H}_{\mu}$,
b) $e_{1}^{\perp} \tilde{H}_{\mu}=\sum_{\mu \rightarrow \nu} c_{\mu \nu} \tilde{H}_{\nu}$, 
and their corresponding summation formulas (see $[6,7]$ for proofs)

$$
\begin{aligned}
& \sum_{\nu \rightarrow \mu} c_{\mu \nu}(q, t)\left(T_{\mu} / T_{\nu}\right)^{k}= \begin{cases}\frac{t q}{M} h_{k+1}\left[D_{\mu}(q, t) / t q\right] & \text { if } k \geq 1, \\
B_{\mu}(q, t) & \text { if } k=0,\end{cases} \\
& \sum_{\mu \leftarrow \nu} d_{\mu \nu}(q, t)\left(T_{\mu} / T_{\nu}\right)^{k}= \begin{cases}(-1)^{k-1} e_{k-1}\left[D_{\nu}(q, t)\right] & \text { if } k \geq 1, \\
1 & \text { if } k=0 .\end{cases}
\end{aligned}
$$

Here $\nu \rightarrow \mu$ simply means that the sum is over $\nu$ 's obtained from $\mu$ by removing a corner cell and $\mu \leftarrow \nu$ means that the sum is over $\mu$ 's obtained from $\nu$ by adding a corner cell.

It will be useful to know that these two Pieri coefficients are related by the identity

$$
d_{\mu \nu}=M d_{\mu \nu} \frac{w_{\nu}}{w_{\mu}} .
$$

In these notes we will make extensive use of the operators $\Delta_{F}$ defined for any symmetric function $F$ by setting for all $\mu$

$$
\Delta_{F} \tilde{H}_{\mu}=F\left[B_{\mu}\right] \tilde{H}_{\mu} .
$$

This given, we should note that the identity in (2.28) has the following surprising consequence

Proposition 2.2. For all polynomials $P \in \Lambda^{=n}$ we have

$$
\left\langle\Delta_{e_{r}} P, h_{n}\right\rangle=\left\langle P, e_{r} h_{n-r}\right\rangle .
$$

Proof. It is sufficient to verify (2.38) for the Macdonald basis $\left\{\tilde{H}_{\mu}\right\}_{\mu}$. But then (2.37) and (2.28) give

$$
\left\langle\Delta_{e_{r}} \tilde{H}_{\mu}, h_{n}\right\rangle=e_{r}\left[B_{\mu}\right]\left\langle\tilde{H}_{\mu}, h_{n}\right\rangle=e_{r}\left[B_{\mu}\right]=\left\langle\tilde{H}_{\mu}, e_{r} h_{n-r}\right\rangle .
$$

The following Macdonald polynomial expansions will occur in many parts of our exposition

Proposition 2.3. For all $n \geq 1$ we have

$$
\begin{aligned}
& \text { a) } e_{n}\left[\frac{X}{M}\right]=\sum_{\mu \vdash n} \frac{\tilde{H}_{\mu}[X ; q, t]}{w_{\mu}} \\
& \text { b) } h_{k}\left[\frac{X}{M}\right] e_{n-k}\left[\frac{X}{M}\right]=\sum_{\mu \vdash n} \frac{e_{k}\left[B_{\mu}\right] \tilde{H}_{\mu}[X ; q, t]}{w_{\mu}}
\end{aligned}
$$




$$
\begin{aligned}
& \text { c) } h_{n}\left[\frac{X}{M}\right]=\sum_{\mu \vdash n} \frac{T_{\mu} \tilde{H}_{\mu}[X ; q, t]}{w_{\mu}} \\
& \text { d) }(-1)^{n-1} p_{n}=\left(1-t^{n}\right)\left(1-q^{n}\right) \sum_{\mu \vdash n} \frac{\Pi_{\mu} \tilde{H}_{\mu}[X ; q, t]}{w_{\mu}}
\end{aligned}
$$

and more generally for $\lambda \vdash n$

$$
s_{\lambda}\left[\frac{X}{M}\right]=\sum_{\mu \vdash n} \frac{\tilde{H}_{\mu}[X]}{w_{\mu}} \tilde{K}_{\lambda^{\prime}, \mu}(q, t)
$$

with the $\tilde{K}_{\lambda, \mu}(q, t)$ the coefficients appearing in the Schur function expansion

$$
\tilde{H}_{\mu}[X]=\sum_{\lambda \vdash n} s_{\lambda}[X] \tilde{K}_{\lambda \mu}(q, t)
$$

Proof. Note first that (2.39) a) and c) are the particular cases $k=0$ and $k=n$ of $(2.39) \mathrm{b})$. Now for any symmetric function $F \in \Lambda^{=n}$ we have

$$
F\left[\frac{X}{M}\right]=\sum_{\mu \vdash n} \frac{\tilde{H}_{\mu}}{w_{\mu}}\left\langle\tilde{H}_{\mu}, F\right\rangle_{*}=\sum_{\mu \vdash n} \frac{\tilde{H}_{\mu}}{w_{\mu}}\left\langle\tilde{H}_{\mu}, \omega F\right\rangle .
$$

This given, (2.39) b) follows by setting $F=h_{k} e_{n-k}$ and using (2.3), while (2.41) follows by setting $F=S_{\lambda}$ and using (2.40). For (2.39) d) we note that (2.18) with $Y \rightarrow 1-z$ and (2.24) give

$$
e_{n}\left[\frac{X(1-u)}{M}\right]=\sum_{\mu \vdash n} \frac{\tilde{H}_{\mu}[X]}{w_{\mu}} \prod_{\mu}\left(1-u t^{l^{\prime}} q^{a^{\prime}}\right) .
$$

Since

$$
\left(1-t^{n}\right)\left(1-q^{n}\right) e_{n}\left[\frac{X(1-u)}{M}\right]=(-1)^{n-1} p_{n}[X] \frac{1-u^{n}}{n}+O\left((1-u)^{2}\right) .
$$

(2.39) d) is obtained by dividing out the common factor $1-u$ from both sides of (2.43) and setting $u=1$.

A Macdonald polynomial tool kit would not be complete without the following truly remarkable identity established as Theorem I.2 in [6]. This identity is gravid with combinatorial implications and has provided the crucial step in the proof a variety of identities in Macdonald polynomial Theory. We will see that it plays a crucial role here as well. 
Theorem 2.4. For any symmetric function $f$ and any partition $\mu$ we have

$$
\left\langle f, \tilde{H}_{\mu}[X+1 ; q, t]\right\rangle_{*}=\left.\nabla^{-1}(f[X-\epsilon])\right|_{X \rightarrow D_{\mu}} .
$$

The reader is referred to [6] for a proof. Here we will use (2.44) in the following special form.

Proposition 2.5. For all $f \in \Lambda^{=k}$ and $\mu \vdash n$ we have

$$
\left\langle f h_{n-k}, \tilde{H}_{\mu}\right\rangle=\left.\nabla^{-1}\left(\omega f^{*}[X-\epsilon]\right)\right|_{X \rightarrow D_{\mu}} .
$$

Proof. The Schur function addition formula

$$
s_{\lambda}[X+Y]=\sum_{\mu \subseteq \mu} s_{\lambda / \mu}[X] s_{\mu}[Y]
$$

implies that for any symmetric polynomial $P[X]$ we have

$$
P[X+Y]=\sum_{\mu} s_{\mu}[Y] S_{\mu}^{\perp} P[X] .
$$

When $Y$ consists of a single letter $y$ this reduces to

$$
P[X+y]=\sum_{m \geq 0} y^{m} h_{m}^{\perp} P[X] .
$$

Now with $f$ replaced by $\omega f^{*}(2.44)$ becomes

$$
\left\langle f, H_{\mu}[X+1]\right\rangle=\left.\nabla^{-1}\left(\omega f^{*}[X-\epsilon]\right)\right|_{X \rightarrow D_{\mu}} .
$$

But then (2.45) for $y=1$ gives

$$
\sum_{m \geq 0}\left\langle f, h_{m}^{\perp} \tilde{H}_{\mu}\right\rangle=\left.\nabla^{-1}\left(\omega f^{*}[X-\epsilon]\right)\right|_{X \rightarrow D_{\mu}} .
$$

But if $f$ is homogeneous of degree $k$ and $\mu \vdash n$ all the summands vanish except for $m=n-k$ reducing this identity to

$$
\left\langle f, h_{n-k}^{\perp} \tilde{H}_{\mu}\right\rangle=\left.\nabla^{-1}\left(\omega f^{*}[X-\epsilon]\right)\right|_{X \rightarrow D_{\mu}}
$$

which is only another way of writing (2.45). 
To illustrate the power of this identity we will use it to derive the following expansion result which we will need in the next section.

\section{Proposition 2.6.}

$$
h_{k}\left[\frac{X}{1-q}\right] e_{n-k}\left[\frac{X}{M}\right]=\sum_{\mu \vdash n} \frac{\tilde{H}_{\mu}[X]}{w_{\mu}} \sum_{r=1}^{k}\left[\begin{array}{l}
k-1 \\
r-1
\end{array}\right]_{q} q^{\left(\begin{array}{c}
r \\
2
\end{array}\right)+r-k r}(-1)^{k-r} h_{r}\left[\frac{M B_{\mu}}{1-q}\right] .
$$

Proof. Using the expansion formula in (2.42) we get

$$
\begin{aligned}
h_{k}\left[\frac{X}{1-q}\right] e_{n-k}\left[\frac{X}{M}\right] & =\sum_{\mu \vdash n} \frac{\tilde{H}_{\mu}[X]}{w_{\mu}}\left\langle\tilde{H}_{\mu}[X], h_{k}\left[\frac{X}{1-q}\right] e_{n-k}\left[\frac{X}{M}\right]\right\rangle_{*} \\
& =\sum_{\mu \vdash n} \frac{\tilde{H}_{\mu}[X]}{w_{\mu}}\left\langle\tilde{H}_{\mu}[X], e_{k}[(1-t) X] h_{n-k}[X]\right\rangle .
\end{aligned}
$$

Now the identity in $(2.45)$, with $f=e_{k}[(1-t) X]$ gives

$$
\left\langle\tilde{H}_{\mu}, e_{k}[(1-t) X] h_{n-k}\right\rangle=\left.\nabla^{-1}\left(\omega f^{*}[X-\epsilon]\right)\right|_{X \rightarrow D_{\mu}}=\left.\nabla^{-1} h_{k}\left[\frac{X-\epsilon}{1-q}\right]\right|_{X \rightarrow D_{\mu}} .
$$

Next note that we have

$$
\begin{aligned}
\nabla^{-1} h_{k}\left[\frac{X-\epsilon}{1-q}\right] & =\nabla^{-1} \sum_{s=0}^{k} h_{k-s}\left[\frac{X}{1-q}\right] h_{s}\left[\frac{-\epsilon}{1-q}\right]=\sum_{s=0}^{k} q^{-\left(\begin{array}{c}
k-s \\
2
\end{array}\right)} h_{k-s}\left[\frac{X}{1-q}\right] e_{s}\left[\frac{1}{1-q}\right] \\
& =\sum_{s=0}^{k} q^{-\left(\begin{array}{c}
k-s \\
2
\end{array}\right)+\left(\begin{array}{c}
s \\
2
\end{array}\right)} h_{k-s}\left[\frac{X}{1-q}\right] \frac{1}{(q, q)_{s}} .
\end{aligned}
$$

Since $-2\left(\begin{array}{c}k-s \\ 2\end{array}\right)+2\left(\begin{array}{c}s \\ 2\end{array}\right)=-k^{2}+2 k s+k-2 s$ we may write

$$
\begin{aligned}
\left.\nabla^{-1} h_{k}\left[\frac{X-\epsilon}{1-q}\right]\right|_{X \rightarrow D_{\mu}} & =\sum_{s=0}^{k} q^{-\left(\begin{array}{c}
k \\
2
\end{array}\right)+k s-s} h_{k-s}\left[\frac{M B_{\mu}-1}{1-q}\right] \frac{1}{(q, q)_{s}} \\
& =\sum_{s=0}^{k} \frac{q^{-\left(\begin{array}{c}
k \\
2
\end{array}\right)+k s-s}}{(q, q)_{s}} \sum_{r=0}^{k-s} h_{r}\left[(1-t) B_{\mu}\right] h_{k-s-r}\left[\frac{-1}{1-q}\right] \\
& =\sum_{s=0}^{k} \frac{q^{-\left(\begin{array}{c}
k \\
2
\end{array}\right)+k s-s}}{(q, q)_{s}} \sum_{r=0}^{k-s} h_{r}\left[(1-t) B_{\mu}\right](-1)^{k-s-r} \frac{q^{\left(k^{k-s-r}\right)}}{(q, q)_{k-s-r}}
\end{aligned}
$$


and since $\left(\begin{array}{c}k-s-r \\ 2\end{array}\right)-\left(\begin{array}{c}k \\ 2\end{array}\right)+k s-s=\left(\begin{array}{c}s \\ 2\end{array}\right)-r k+s r+\left(\begin{array}{c}r+1 \\ 2\end{array}\right)$ we get

$$
\begin{aligned}
\nabla^{-1} & \left.h_{k}\left[\frac{X-\epsilon}{1-q}\right]\right|_{X \rightarrow D_{\mu}} \\
& =\sum_{r=0}^{k} \frac{(-1)^{k-r} q^{\left(\begin{array}{c}
r+1 \\
2
\end{array}\right)-r k}}{(q, q)_{k-r}} h_{r}\left[(1-t) B_{\mu}\right] \sum_{s=0}^{k-r}\left[\begin{array}{c}
k-r \\
s
\end{array}\right]_{q} q^{\left(\begin{array}{c}
s \\
2
\end{array}\right)(-1)^{s}\left(q^{r}\right)^{s}}
\end{aligned}
$$

(by the $q$-binomial Theorem)

$$
\begin{aligned}
& =\sum_{r=0}^{k} \frac{(-1)^{k-r} q_{2}^{\left(\begin{array}{c}
r+1 \\
2
\end{array}\right)-r k}}{(q, q)_{k-r}} h_{r}\left[(1-t) B_{\mu}\right]\left(1-q^{r}\right)\left(1-q^{r+1}\right) \cdots\left(1-q^{r+k-r-1}\right) \\
& =\sum_{r=1}^{k}\left[\begin{array}{c}
k-1 \\
r-1
\end{array}\right]_{q}(-1)^{k-r} q^{\left(\begin{array}{c}
r+1 \\
2
\end{array}\right)-r k} h_{r}\left[(1-t) B_{\mu}\right]
\end{aligned}
$$

which combined with (2.49) and (2.48) completes our proof of (2.47).

\section{Auxiliary Macdonald Polynomial identities}

Recall the polynomials $E_{n k}$ were introduced in [3] by means of the following expansion

$$
e_{n}\left[X \frac{1-z}{1-q}\right]=\sum_{k=1}^{n} \frac{(z, q)_{k}}{(q, q)_{k}} E_{n k}
$$

In [10] Haglund establishes the following

Proposition 3.1. For all $1 \leq k \leq n$ we have

$$
\nabla E_{n k}=t^{n-k}\left(1-q^{k}\right) \Psi h_{k}\left[\frac{X}{1-q}\right] h_{n-k}\left[\frac{X}{M}\right]
$$

where $\Psi$ is the symmetric function operator defined by setting for all $\mu$

$$
\Psi \tilde{H}_{\mu}=\Pi_{\mu} \tilde{H}_{\mu} .
$$

The proof of (3.2) in [10] relies on a variety of auxiliary identities established in [3] and [10]. As a result it is quite lengthy when all the details are included. Fortunately we have been able to reduce it to what we believe are the bare essentials. However since it is still quite technical we will postpone its proof to the end of this section. For the next auxiliary identities, expressed as a series of propositions, our proofs should be considerably simpler than in the original work. 
Proposition 3.2. For $\lambda \vdash m$

$$
\left\langle\Delta_{S_{\lambda}} \nabla E_{n k}, h_{n}\right\rangle=t^{n-k}\left(1-q^{k}\right) \sum_{\nu \vdash m} \frac{\Pi_{\nu}}{w_{\nu}} h_{k}\left[(1-t) B_{\nu}\right] h_{n-k}\left[B_{\nu}\right] \tilde{K}_{\lambda^{\prime}, \nu}
$$

Proof. Using (3.2) the left-hand side of (3.3) can be rewritten as

$$
\begin{aligned}
\left\langle\Delta_{s_{\lambda}} \nabla E_{n k}, h_{n}\right\rangle & =t^{n-k}\left(1-q^{k}\right)\left\langle\Delta_{s_{\lambda}} \Psi h_{k}\left[\frac{X}{1-q}\right] h_{n-k}\left[\frac{X}{M}\right], h_{n}\right\rangle \\
& =t^{n-k}\left(1-q^{k}\right)\left\langle\Delta_{s_{\lambda}} \Psi h_{k}\left[\frac{X}{1-q}\right] h_{n-k}\left[\frac{X}{M}\right], e_{n}^{*}\right\rangle_{*} \\
& =t^{n-k}\left(1-q^{k}\right)\left\langle h_{k}\left[\frac{X}{1-q}\right] h_{n-k}\left[\frac{X}{M}\right], \Psi \Delta_{s_{\lambda}} e_{n}^{*}\right\rangle_{*} .
\end{aligned}
$$

However the relations in (2.39) a) and (2.40), namely

$$
\begin{aligned}
\text { a) } e_{n}^{*}[X]=\sum_{\mu \vdash n} \frac{\tilde{H}_{\mu}[X]}{w_{\mu}}, \quad \text { b) } s_{\lambda}^{*}[X]=\sum_{\mu \vdash n} \frac{\tilde{H}_{\mu}[X]}{w_{\mu}} \tilde{K}_{\lambda^{\prime} \mu} \\
\Psi \Delta_{s_{\lambda}} e_{n}^{*}[X]=\sum_{\mu \vdash n} \frac{\Pi_{\mu} \tilde{H}_{\mu}[X]}{w_{\mu}} s_{\lambda}\left[\frac{M B_{\mu}}{M}\right] \\
\text { (by (3.5) b) })=\sum_{\mu \vdash n} \frac{\Pi_{\mu} \tilde{H}_{\mu}[X]}{w_{\mu}} \sum_{\nu \vdash m} \frac{\tilde{H}_{\nu}\left[M B_{\mu}\right]}{w_{\nu}} \tilde{K}_{\lambda^{\prime}, \nu}
\end{aligned}
$$

(Using reciprocity as in (2.12)) $=\sum_{\mu \vdash n} \frac{\tilde{H}_{\mu}[X]}{w_{\mu}} \sum_{\nu \vdash m} \frac{\Pi_{\nu} \tilde{H}_{\mu}\left[M B_{\nu}\right]}{w_{\nu}} \tilde{K}_{\lambda^{\prime}, \nu}$

$$
=\sum_{\nu \vdash m} \frac{\Pi_{\nu}}{w_{\nu}} \tilde{K}_{\lambda^{\prime}, \nu} \sum_{\mu \vdash n} \frac{\tilde{H}_{\mu}[X]}{w_{\mu}} \tilde{H}_{\mu}\left[M B_{\nu}\right] .
$$

Using (3.6) in (3.4) yields

$$
\begin{aligned}
& \left\langle\Delta_{s_{\lambda}} \nabla E_{n k}, h_{n}\right\rangle \\
& =t^{n-k}\left(1-q^{k}\right) \sum_{\nu \vdash j} \frac{\Pi_{\nu}}{w_{\nu}} \tilde{K}_{\lambda^{\prime}, \nu}\left\langle h_{k}\left[\frac{X}{1-q}\right] h_{n-k}\left[\frac{X}{M}\right], \sum_{\mu \vdash n} \frac{\tilde{H}_{\mu}[X]}{w_{\mu}} \tilde{H}_{\mu}\left[M B_{\nu}\right]\right\rangle_{*} \\
& =t^{n-k}\left(1-q^{k}\right) \sum_{\nu \vdash j} \frac{\Pi_{\nu}}{w_{\nu}} \tilde{K}_{\lambda^{\prime}, \nu}\left(\left\langle h_{k}\left[\frac{X}{1-q}\right] h_{n-k}\left[\frac{X}{M}\right], \sum_{\mu \vdash n} \frac{\tilde{H}_{\mu}[X]}{w_{\mu}} \tilde{H}_{\mu}[Y]\right\rangle_{*}\right)_{Y \rightarrow M B_{\nu}} \\
& =t^{n-k}\left(1-q^{k}\right) \sum_{\nu \vdash j} \frac{\Pi_{\nu}}{w_{\nu}}\left(\tilde{K}_{\lambda^{\prime}, \nu} h_{k}\left[\frac{Y}{1-q}\right] h_{n-k}\left[\frac{Y}{M}\right]\right)_{Y \rightarrow M B_{\nu}} .
\end{aligned}
$$


In particular the identity in (4) has the following specialization

\section{Proposition 3.3.}

$$
\left\langle\Delta_{h_{j}} E_{n k}, e_{n}\right\rangle=t^{n-k}\left(1-q^{k}\right) \sum_{\nu \vdash j} \frac{T_{\nu} \Pi_{\nu}}{w_{\nu}} h_{k}\left[(1-t) B_{\nu}\right] h_{n-k}\left[B_{\nu}\right] .
$$

Proof. Letting $\lambda=(j)$ in (3.3) we see that $\tilde{K}_{\lambda^{\prime}, \mu}=T_{\mu}$ and then (3.7) follows from Proposition 2.2.

Proposition 3.4. For all $n, k \geq 1$ the symmetric polynomial $e_{n}\left[X \frac{1-q^{k}}{1-q}\right]$ has the following Macdonald polynomial expansion

$$
e_{n}\left[X \frac{1-q^{k}}{1-q}\right]=\left(1-q^{k}\right) \sum_{\mu \vdash n} \frac{\tilde{H}_{\mu}[X] \Pi_{\mu} h_{k}\left[(1-t) B_{\mu}\right]}{w_{\mu}} .
$$

Proof. The Macdonald "Cauchy" formula in (2.19) gives

$$
\begin{aligned}
e_{n}\left[X \frac{1-q^{k}}{1-q}\right] & =e_{n}\left[X \frac{(1-t)\left(1-q^{k}\right)}{M}\right] \\
& =\sum_{\mu \vdash n} \frac{\tilde{H}_{\mu}[X] \tilde{H}_{\mu}\left[(1-t)\left(1-q^{k}\right)\right]}{w_{\mu}} .
\end{aligned}
$$

But

$$
\begin{aligned}
\tilde{H}_{\mu}\left[(1-t)\left(1-q^{k}\right)\right] & =\tilde{H}_{\mu}\left[\frac{M\left(1-q^{k}\right)}{1-q}\right]=\tilde{H}_{\mu}\left[M B_{k}\right] \\
(\text { by reciprocity }) & =\Pi_{\mu} \frac{\tilde{H}_{k}\left[M B_{\mu}\right]}{\Pi_{(k)}}=\Pi_{\mu} \frac{h_{k}\left[(1-t) B_{\mu}\right]}{\Pi_{(k)}}(q, q)_{k} \\
& =\left(1-q^{k}\right) \Pi_{\mu} h_{k}\left[(1-t) B_{\mu}\right] .
\end{aligned}
$$

Using this in (3.9) gives (3.8) as desired.

By combining the last two propositions we obtain

\section{Proposition 3.5.}

$$
\left\langle\Delta_{h_{j}} E_{n k}, e_{n}\right\rangle=t^{n-k}\left\langle\Delta_{h_{n-k}} e_{j}\left[X \frac{1-q^{k}}{1-q}\right], e_{j}\right\rangle .
$$

Proof. Applying the operator $\Delta_{h_{n-k}}$ to (3.8) with $n \rightarrow j$ we get

$$
\Delta_{h_{n-k}} e_{j}\left[X \frac{1-q^{k}}{1-q}\right]=\left(1-q^{k}\right) \sum_{\nu \vdash j} \frac{\tilde{H}_{\nu}[X] \Pi_{\nu} h_{k}\left[(1-t) B_{\nu}\right] h_{n-k}\left[B_{\nu}\right]}{w_{\nu}} .
$$


Thus, since $\left\langle\tilde{H}_{\nu}, e_{j}\right\rangle=T_{\nu}$, we also have

$$
\left\langle\Delta_{h_{n-k}} e_{j}\left[X \frac{1-q^{k}}{1-q}\right], e_{j}\right\rangle=\left(1-q^{k}\right) \sum_{\nu \vdash j} \frac{T_{\nu} \Pi_{\nu}}{w_{\nu}} h_{k}\left[(1-t) B_{\nu}\right] h_{n-k}\left[B_{\nu}\right]
$$

But now a simple comparison of the right-hand sides of (3.7) and (3.11) yields that (3.7) and (3.10) are in fact equivalent.

The next identity is the contents of Corollary 2.9 in Haglund's q,tSchröder paper. It is stated as follows

Proposition 3.6. For any positive integers $m, n$ and any $P \in \Lambda^{n}$. we have

$$
\left\langle\Delta_{e_{m-1}} e_{n}, P\right\rangle=\left\langle\Delta_{\omega P} e_{m}, s_{m}\right\rangle
$$

Proof. The operator $\Delta_{e_{m-1}}$ applied to both sides of (2.32) gives

$$
\Delta_{e_{m-1}} e_{n}=\sum_{\mu \vdash n} \frac{M B_{\mu} \Pi_{\mu} \tilde{H}_{\mu}(x ; q, t)}{w_{\mu}} e_{m-1}\left[B_{\mu}\right] .
$$

Using again (2.32) with $n \rightarrow m$ we also have

$$
\Delta_{\omega P} e_{m}=\sum_{\alpha \vdash m} \frac{M B_{\alpha} \Pi_{\alpha} \tilde{H}_{\alpha}(x ; q, t)}{w_{\alpha}}(\omega P)\left[B_{\alpha}\right] .
$$

Using (3.13) and (3.14) we get the explicit form of (3.12), which is

$$
\sum_{\mu \vdash n} \frac{M B_{\mu} \Pi_{\mu} e_{m-1}\left[B_{\mu}\right]}{w_{\mu}}\left\langle\tilde{H}_{\mu}, P\right\rangle=\sum_{\alpha \vdash m} \frac{M B_{\alpha} \Pi_{\alpha}}{w_{\alpha}}(\omega P)\left[B_{\alpha}\right] .
$$

This given, our idea, as in Haglund's paper, is to establish (3.15) by checking its validity when $P$ varies among all the members of a symmetric function basis. It turns out that a simpler proof of (3.12) is obtained by testing (3.15) with the modified Macdonald basis

$$
\left\{\frac{\omega \tilde{H}_{\gamma}[M X ; q, t]}{w_{\gamma}}\right\}_{\gamma}
$$

The source of the simplification is due to the fact that this basis is precisely the Hall scalar product dual of the Macdonald basis $\left\{\tilde{H}_{\gamma}[X]\right\}_{\gamma}$. Using this fact, putting $P=\frac{\omega \tilde{H}_{\gamma}[M X]}{w_{\gamma}}$ in (3.15) gives 


$$
\frac{M B_{\gamma} \Pi_{\gamma} e_{m-1}\left[B_{\gamma}\right]}{w_{\gamma}}=\sum_{\alpha \vdash m} \frac{M B_{\alpha} \Pi_{\alpha}}{w_{\alpha}} \frac{\tilde{H}_{\gamma}\left[M B_{\alpha}\right]}{w_{\gamma}} .
$$

Carrying out the simplifications this may be rewritten as

$$
B_{\gamma} e_{m-1}\left[B_{\gamma}\right]=\sum_{\alpha \vdash m} \frac{B_{\alpha} \Pi_{\alpha}}{w_{\alpha}} \frac{\tilde{H}_{\gamma}\left[M B_{\alpha}\right]}{\Pi_{\gamma}}
$$

and Macdonald reciprocity reduces this to

$$
B_{\gamma} e_{m-1}\left[B_{\gamma}\right]=\sum_{\alpha \vdash m} \frac{B_{\alpha}}{w_{\alpha}} \tilde{H}_{\alpha}\left[M B_{\gamma}\right] .
$$

Now we may rewrite (2.34) with $k=0$, as

$$
B_{\alpha}=\sum_{\beta \rightarrow \alpha} c_{\alpha \beta}
$$

Using this in the right-hand side of (3.17) gives

(3.18) $R H S=\sum_{\alpha \vdash m} \frac{\tilde{H}_{\alpha}\left[M B_{\gamma}\right]}{w_{\alpha}} \sum_{\beta \rightarrow \alpha} c_{\alpha \beta}=\sum_{\beta \vdash m-1} \frac{1}{w_{\beta}} \sum_{\alpha \leftarrow \beta} \tilde{H}_{\alpha}\left[M B_{\gamma}\right] c_{\alpha \beta} \frac{w_{\beta}}{w_{\alpha}}$.

Next we use (2.36) to get

and (3.18) becomes

$$
c_{\alpha \beta} \frac{w_{\beta}}{w_{\alpha}}=\frac{1}{M} d_{\alpha \beta}
$$

$$
\begin{aligned}
R H S & =\frac{1}{M} \sum_{\beta \vdash m-1} \frac{1}{w_{\beta}} \sum_{\alpha \leftarrow \beta} \tilde{H}_{\alpha}\left[M B_{\gamma}\right] d_{\alpha \beta} \\
& =\frac{1}{M} \sum_{\beta \vdash m-1} \frac{M B_{\gamma} \tilde{H}_{\alpha}\left[M B_{\gamma}\right]}{w_{\beta}} \\
& =B_{\gamma} \sum_{\beta \vdash m-1} \frac{\tilde{H}_{\alpha}\left[M B_{\gamma} ; q, t\right]}{w_{\beta}} \\
(\text { by }(2.39) \text { a) }) & =B_{\gamma} e_{m-1}\left[\frac{M B_{\gamma}}{M}\right]=B_{\gamma} e_{m-1}\left[B_{\gamma}\right]
\end{aligned}
$$

proving (3.16) as desired. 
Next, we give the proof of Proposition 3.1. To begin, it will be convenient to set

$$
L H S=\nabla E_{n k} \quad \text { and } \quad R H S=t^{n-k}\left(1-q^{k}\right) \Psi h_{k}\left[\frac{X}{1-q}\right] h_{n-k}\left[\frac{X}{M}\right] .
$$

We are to show that

$$
L H S=R H S .
$$

It turns out that it is easier to show that we have

$$
\downarrow L H S=\downarrow R H S
$$

where " $\downarrow$ " denotes the symmetric polynomial operator defined by setting, for any symmetric function $F[X ; q, t]$ with coefficients in $\mathbb{Q}[q, t]$,

$$
\downarrow F[X ; q, t]=\omega F[X ; 1 / q, 1 / t] .
$$

Since " $\downarrow$ " is clearly an involution we see that (3.20) is equivalent to (3.19). To carry this out we need the following auxiliary identities

Lemma 3.7. For all $\mu \vdash n$ we have
a) $\downarrow \nabla \downarrow=\nabla^{-1}$,
b) $\downarrow \Psi \downarrow=(-1)^{n-1} \nabla^{-1} \Psi$.

Proof. Recall that by definition
a) $\nabla \tilde{H}_{\mu}[X ; q, t]=T_{\mu} \tilde{H}_{\mu}[X ; q, t]$,
b) $\Psi \tilde{H}_{\mu}[X ; q, t]=\Pi_{\mu} \tilde{H}_{\mu}[X ; q, t]$.

Applying " $\downarrow$ " to both sides of (3.23) a) gives

$$
\downarrow \nabla \downarrow \omega \tilde{H}_{\mu}[X ; 1 / q, 1 / t]=\frac{1}{T_{\mu}} \omega \tilde{H}_{\mu}[X ; 1 / q, 1 / t] .
$$

Recalling the Macdonald identity

$$
T_{\mu} \omega \tilde{H}_{\mu}[X ; 1 / q, 1 / t]=\tilde{H}_{\mu}[X ; q, t]
$$

we see that multiplying both sides of (3.24) by $T_{\mu}$ gives

$$
\downarrow \nabla \downarrow \tilde{H}_{\mu}[X ; q, t]=\frac{1}{T_{\mu}} \tilde{H}_{\mu}[X ; q, t] .
$$


This proves (3.22) a). Similarly (3.23) b) gives

$$
\downarrow \Psi \downarrow \omega \tilde{H}_{\mu}[X ; 1 / q, 1 / t]=\Pi_{\mu}(1 / q, 1 / t) \omega \tilde{H}_{\mu}[X ; 1 / q, 1 / t] .
$$

However, for $\mu \vdash n$ we have

$$
\Pi_{\mu}(1 / q, 1 / t)=\prod_{c \in \mu /(o, o)}\left(1-t^{-l^{\prime}} q^{-a^{\prime}}\right)=\frac{(-1)^{n-1}}{T_{\mu}} \Pi_{\mu}(q, t)
$$

and another use of (3.25) gives

$$
\downarrow \Psi \downarrow \tilde{H}_{\mu}[X ; q, t]=\frac{(-1)^{n-1}}{T_{\mu}} \Pi_{\mu} \tilde{H}_{\mu}[X ; q, t]=(-1)^{n-1} \Psi \nabla^{-1} \tilde{H}_{\mu}[X ; q, t] .
$$

This proves (3.22) b).

Now it is well known and easy to show (see [3]) that the expansion of any polynomial $P(z)$ in terms of the Pokhammer polynomials

$$
(z ; q)_{k}=(1-x)(1-x q) \cdots\left(1-x q^{k-1}\right)
$$

is given by the formula

$$
P(z)=\sum_{k \geq 0} \frac{(z ; q)_{k}}{(q ; q)_{k}} q^{k} \sum_{r=0}^{k}\left[\begin{array}{l}
k \\
r
\end{array}\right]_{q} q^{\left(\begin{array}{c}
r \\
2
\end{array}\right)(-1)^{r} P\left(1 / q^{r}\right) .}
$$

Thus from the definition in (3.1) we immediately derive that

$$
E_{n k}[X ; q]=q^{k} \sum_{r=0}^{k}\left[\begin{array}{l}
k \\
r
\end{array}\right]_{q} q^{\left(\begin{array}{c}
r \\
2
\end{array}\right)}(-1)^{r} e_{n}\left[X \frac{1-q^{-r}}{1-q}\right] .
$$

Thus

$$
L H S=\nabla q^{k} \sum_{r=0}^{k}\left[\begin{array}{l}
k \\
r
\end{array}\right]_{q} q^{\left(\begin{array}{l}
r \\
2
\end{array}\right)(-1)^{r} e_{n}\left[X \frac{1-q^{-r}}{1-q}\right]}
$$

and since

$$
\begin{aligned}
\downarrow\left[\begin{array}{l}
k \\
r
\end{array}\right]_{q} & =\frac{(1-1 / q)\left(1-1 / q^{2}\right) \cdots\left(1-1 / q^{k}\right)}{(1-1 / q)\left(1-1 / q^{2}\right) \cdots\left(1-1 / q^{r}\right)(1-1 / q)\left(1-1 / q^{2}\right) \cdots\left(1-1 / q^{k-r}\right)} \\
& =\left[\begin{array}{l}
k \\
r
\end{array}\right]_{q} q^{r^{2}-k r}
\end{aligned}
$$


using (3.22) a), the definition in (3.21) gives

$$
\begin{aligned}
\downarrow L H S & =\nabla^{-1} q^{-k} \sum_{r=0}^{k}\left[\begin{array}{l}
k \\
r
\end{array}\right]_{q} q^{r^{2}-k r-\left(\begin{array}{c}
r \\
2
\end{array}\right)(-1)^{r} h_{n}\left[X \frac{1-q^{r}}{1-1 / q}\right]} \\
& =\nabla^{-1} q^{n-k} \sum_{r=0}^{k}\left[\begin{array}{l}
k \\
r
\end{array}\right]_{q} q^{r^{2}-k r-\left(\begin{array}{c}
r \\
2
\end{array}\right)(-1)^{r} h_{n}\left[X \frac{1-q^{r}}{q-1}\right]} \\
& =\nabla^{-1} q^{n-k} \sum_{r=0}^{k}\left[\begin{array}{l}
k \\
r
\end{array}\right]_{q} q^{\left(\begin{array}{c}
r \\
2
\end{array}\right)+r-k r}(-1)^{n-r} e_{n}\left[X \frac{1-q^{r}}{1-q}\right] .
\end{aligned}
$$

On the other hand (3.21) and (3.22) b) give

$$
\begin{aligned}
\downarrow R H S & =t^{k-n}\left(1-q^{-k}\right)(-1)^{n-1} \nabla^{-1} \Psi e_{k}\left[\frac{X}{1-1 / q}\right] e_{n-k}\left[\frac{X q t}{M}\right] \\
& =t^{k-n}\left(q^{k}-1\right)(-1)^{n-1} \nabla^{-1} \Psi e_{k}\left[\frac{X}{q-1}\right] e_{n-k}\left[\frac{X}{M}\right] q^{n-k} t^{n-k} \\
& =q^{n-k}\left(1-q^{k}\right)(-1)^{n-k} \nabla^{-1} \Psi h_{k}\left[\frac{X}{1-q}\right] e_{n-k}\left[\frac{X}{M}\right] .
\end{aligned}
$$

Thus (3.20) reduces to

$$
\sum_{r=0}^{k}\left[\begin{array}{l}
k \\
r
\end{array}\right]_{q} q^{\left(\begin{array}{l}
r \\
2
\end{array}\right)+r-k r}(-1)^{n-r} \Psi^{-1} e_{n}\left[X \frac{1-q^{r}}{1-q}\right]=\left(1-q^{k}\right)(-1)^{n-k} h_{k}\left[\frac{X}{1-q}\right] e_{n-k}\left[\frac{X}{M}\right] .
$$

However, the Macdonald Cauchy formula in (2.19) gives

$$
e_{n}\left[X \frac{1-q^{r}}{1-q}\right]=e_{n}\left[\frac{X(1-t)\left(1-q^{r}\right)}{M}\right]=\sum_{\mu \vdash n} \frac{\tilde{H}_{\mu}[X]}{w_{\mu}} \tilde{H}_{\mu}\left[M \frac{1-q^{r}}{1-q}\right]
$$

Thus

$$
\Psi^{-1} e_{n}\left[X \frac{1-q^{r}}{1-q}\right]=\sum_{\mu \vdash n} \frac{\tilde{H}_{\mu}[X]}{w_{\mu}} \frac{\tilde{H}_{\mu}\left[M \frac{1-q^{r}}{1-q}\right]}{\Pi_{\mu}}
$$

(by $(2.22)$ for $\alpha, \beta \rightarrow \mu, r)=\sum_{\mu \vdash n} \frac{\tilde{H}_{\mu}[X]}{w_{\mu}} \frac{\tilde{H}_{r}\left[M B_{\mu}\right]}{\Pi_{r}}$

$$
=\sum_{\mu \vdash n} \frac{\tilde{H}_{\mu}[X]}{w_{\mu}}\left(1-q^{r}\right) h_{r}\left[\frac{M B_{\mu}}{1-q}\right] .
$$


Here we have used $\tilde{H}_{r}[X]=(q, q)_{r} h_{r}\left[\frac{X}{1-q}\right]$ and $\Pi_{r}=(q, q)_{r-1}$, and (3.27) becomes

$$
\begin{aligned}
& \sum_{\mu \vdash n} \frac{\tilde{H}_{\mu}[X]}{w_{\mu}} \sum_{r=0}^{k}\left[\begin{array}{l}
k \\
r
\end{array}\right]_{q} q^{\left(\begin{array}{c}
r \\
2
\end{array}\right)+r-k r}(-1)^{n-r}\left(1-q^{r}\right) h_{r}\left[\frac{M B_{\mu}}{1-q}\right] \\
& \quad=\left(1-q^{k}\right)(-1)^{n-k} h_{k}\left[\frac{X}{1-q}\right] e_{n-k}\left[\frac{X}{M}\right]
\end{aligned}
$$

which is easily seen to simplify to

$$
\sum_{\mu \vdash n} \frac{\tilde{H}_{\mu}[X]}{w_{\mu}} \sum_{r=1}^{k}\left[\begin{array}{l}
k-1 \\
r-1
\end{array}\right]_{q} q^{\left(\begin{array}{c}
r \\
2
\end{array}\right)+r-k r}(-1)^{k-r} h_{r}\left[\frac{M B_{\mu}}{1-q}\right]=h_{k}\left[\frac{X}{1-q}\right] e_{n-k}\left[\frac{X}{M}\right]
$$

which is precisely the expansion in (2.47). This completes our proof of Proposition 3.1.

\section{The symmetric function side}

The first step in the proof of Theorem 1.1 is given by the following surprising identity

\section{Proposition 4.1.}

$$
\left\langle\nabla e_{n}, h_{j} h_{n-j}\right\rangle=\left\langle\Delta_{h_{j}} e_{n+1-j}, e_{n+1-j}\right\rangle .
$$

Proof. This is obtained in [10] by a magic double use of the identity in (3.12), namely

$$
\left\langle\Delta_{e_{m-1}} e_{n}, P\right\rangle=\left\langle\Delta_{\omega P} e_{m}, s_{m}\right\rangle
$$

Haglund starts by using it with $m-1=n$ and $P=h_{j} h_{n-j}$ obtaining

$$
\left\langle\nabla e_{n}, h_{j} h_{n-j}\right\rangle=\left\langle\Delta_{e_{j} e_{n-j}} e_{n+1}, s_{n+1}\right\rangle=\left\langle\Delta_{e_{n-j}} e_{n+1}, e_{j} h_{n+1-j}\right\rangle
$$

here the last equality follows from (2.38) and the fact that $\Delta_{e_{j} e_{n-j}}=\Delta_{e_{j}} \Delta_{e_{n-j}}$. Using (4.2) again with $n \rightarrow n+1, m \rightarrow n+1-j$ and $P \rightarrow e_{j} h_{n+1-j}$ we get

$$
\begin{aligned}
\left\langle\Delta_{e_{n-j}} e_{n+1}, e_{j} h_{n+1-j}\right\rangle & =\left\langle\Delta_{h_{j} e_{n+1-j}} e_{n-j+1}, s_{n-j+1}\right\rangle \\
& =\left\langle\Delta_{h_{j}} \nabla e_{n-j+1}, e_{n-j+1}\right\rangle .
\end{aligned}
$$

Combining (4.4) and (4.3) gives (4.1) as desired. 
Now note that by setting $z=q$ in (3.1) we derive that

$$
e_{n}=\sum_{k=1}^{n} E_{n k}
$$

Using this with $n \rightarrow n+1-j$ we may rewrite (4.1) as

$$
\left\langle\nabla e_{n}, h_{j} h_{n-j}\right\rangle=\sum_{k=1}^{n+1-j}\left\langle\Delta_{j} E_{n-j+1, k}, e_{n+1-j}\right\rangle
$$

But Theorem 1.2 with $n \rightarrow n+1-j$ asserts that

$$
\begin{aligned}
& \left\langle\Delta_{j} E_{n-j+1, k}, e_{n+1-j}\right\rangle \\
& \quad=\sum_{P F \in \mathcal{P F}_{n+1}(k)} t^{\operatorname{area}(P F)} q^{\operatorname{dinv}(P F)} \chi(\sigma(P F) \in 12 \cdots j \cup \cup j+1 \cdots n+1)
\end{aligned}
$$

where here $\mathcal{P} \mathcal{F}_{n+1}(k)$ denotes the collection of Parking Functions, in the $(n+1) \times(n+1)$ lattice square, with $k$ of the cars $j+1, \ldots, n+1$ in the main diagonal and car $n+1$ in the cell $(1,1)$. This given, using (4.5) with $n \rightarrow n+1-j$ we can convert (4.7) to

$$
\begin{aligned}
& \left\langle\nabla e_{n}, h_{j} h_{n-j}\right\rangle \\
& \quad=\sum_{P F \in \mathcal{P F}_{n+1}^{\prime}} t^{\operatorname{area}(P F)} q^{\operatorname{dinv}(P F)} \chi(\sigma(P F) \in 12 \cdots j \cup \cup j+1 \cdots n+1)
\end{aligned}
$$

where $\mathcal{P} \mathcal{F}_{n+1}$ denotes the collection of Parking Functions in the $(n+1) \times$ $(n+1)$ lattice square, with car $n+1$ in the cell $(1,1)$. Since clearly, car $n+1$ does not contribute any dinv, and in addition, there are no cars above it, $n+1$ can be removed, without loss, along with its column and row, in each term on the right hand side of (4.8) and thereby obtain that

$$
\left\langle\nabla e_{n}, h_{j} h_{n-j}\right\rangle=\sum_{P F \in \mathcal{P} \mathcal{F}_{n}} t^{\operatorname{area}(P F)} q^{\operatorname{dinv}(P F)} \chi(\sigma(P F) \in 12 \cdots j \cup \cup j+1 \cdots n)
$$

which is precisely what Theorem 1.1 does assert.

We are thus reduced to proving Theorem 1.2. As we stated in the introduction, to do this we need only show that both sides of Theorem 1.2 satisfy the same recursion and initial conditions. We will thus begin with the symmetric function side and prove (1.4), that is 
Proposition 4.2. Setting $H_{j, n, k}=\left\langle\Delta_{h_{j}} E_{n k}, e_{n}\right\rangle$ we have for all $j \geq 1$ and $1 \leq k \leq n$

$$
H_{j, n, k}=t^{n-k} \sum_{s=1}^{j}\left[\begin{array}{c}
k+s-1 \\
s
\end{array}\right]_{q} H_{n-k, j, s} .
$$

Proof. Setting $z=q^{k}$ in the defining formula (3.1) with $n \rightarrow j$ we get

$$
e_{j}\left[X \frac{1-q^{k}}{1-q}\right]=\sum_{s=1}^{j} \frac{\left(q^{k}, q\right)_{s}}{(q, q)_{s}} E_{j s} .
$$

This given, (3.10) gives

$$
\begin{aligned}
\left\langle\Delta_{h_{j}} E_{n k}, e_{n}\right\rangle & =t^{n-k}\left\langle\Delta_{h_{n-k}} e_{j}\left[X \frac{1-q^{k}}{1-q}\right], e_{j}\right\rangle \\
& =t^{n-k} \sum_{s=1}^{j} \frac{\left(q^{k}, q\right)_{s}}{(q, q)_{s}}\left\langle\Delta_{h_{n-k}} E_{j s}, e_{j}\right\rangle
\end{aligned}
$$

and the identity

$$
\frac{\left(q^{k}, q\right)_{s}}{(q, q)_{s}}=\frac{\left(1-q^{k}\right) \cdots\left(1-q^{k+s-1}\right)}{(1-1) \cdots\left(1-q^{s}\right)}=\left[\begin{array}{c}
k+s-1 \\
s
\end{array}\right]_{q}
$$

gives (4.10) and completes our proof.

Since the total number of cars decreases by $k$ at each use of the recursion and $k \geq 1$ we will eventually run in to a case where the number of small cars vanishes, thus the following result settles all the base cases.

\section{Proposition 4.3.}

$$
H_{0, n, k}=\left\langle E_{n k}, e_{n}\right\rangle=\left\langle\nabla E_{n k}, h_{n}\right\rangle= \begin{cases}0 & \text { if } 1 \leq k<n \\ 1 & \text { if } k=n\end{cases}
$$

Proof. Note that the first equality in (4.11) is simply due to the fact that $\Delta_{h_{j}}$ reduces to the identity operator for $j=0$. The second equality follows from (2.38) for $r=n$.

To prove the third equality we can make use of Proposition 3.1. In fact, (3.4) with $\Delta_{s_{\lambda}}$ replaced by the identity operator gives

$$
\left\langle\nabla E_{n k}, h_{n}\right\rangle=t^{n-k}\left(1-q^{k}\right)\left\langle h_{k}\left[\frac{X}{1-q}\right] h_{n-k}\left[\frac{X}{M}\right], \Psi e_{n}^{*}\right\rangle_{*} .
$$


On the other hand from from (2.39) a) and d) we derive that

$$
\Psi e_{n}^{*}=\sum_{\mu \vdash n} \frac{\Pi_{\mu} \tilde{H}_{\mu}[X]}{w_{\mu}}=\frac{(-1)^{n-1}}{\left(1-t^{n}\right)\left(1-q^{n}\right)} p_{n} .
$$

Using this in (4.12) immediately gives the first case of (4.11), since for $1 \leq$ $k<n$ the power sum expansion of the product $h_{k}\left[\frac{X}{1-q}\right] h_{n-k}\left[\frac{X}{M}\right]$ cannot contain a term with $p_{n}$. On the other hand for $k=n$ (4.12) becomes

$$
\left\langle\nabla E_{n n}, h_{n}\right\rangle=\left(1-q^{n}\right)\left\langle h_{n}\left[\frac{X}{1-q}\right], \omega p_{n}^{*}\right\rangle_{*}=\left(1-q^{n}\right)\left\langle h_{n}\left[\frac{X}{1-q}\right], p_{n}\right\rangle=1
$$

as desired.

Remark. There is an interesting recursive proof of (4.11) which uses the symmetric function operator $\mathbf{C}_{a}$ defined by setting, for a symmetric function $F[X]$

$$
\mathbf{C}_{a} F[X]=\left.\left(-\frac{1}{q}\right)^{a-1} F\left[X-\frac{1-1 / q}{z}\right] \Omega[z X]\right|_{z^{a}}
$$

In fact it is shown in [8] that the $E_{n k}$ can be recursively obtained from the formula

$$
E_{n k}=\sum_{a=1}^{n-k+1} \mathbf{C}_{a} E_{n-a, k-1}
$$

Using this, the left hand side of (4.11) becomes

$$
\begin{aligned}
\left\langle E_{n k}, e_{n}\right\rangle & =\sum_{a=1}^{n-k+1}\left\langle\mathbf{C}_{a} E_{n-a, k-1}, e_{n}\right\rangle \\
& =\sum_{a=1}^{n-k+1}\left\langle E_{n-a, k-1}, \mathbf{C}_{a}^{*} h_{n}^{*}\right\rangle_{*}
\end{aligned}
$$

with $\mathbf{C}_{a}^{*}$ the $*$-scalar product dual of $\mathbf{C}_{a}$. Now it shown in [8] that $\mathbf{C}_{a}^{*}$ acts on a symmetric function $F[X]$ according to the plethystic formula

$$
\mathbf{C}_{a}^{*} P[X]=\left.\left(\frac{-1}{q}\right)^{a-1} P\left[X-\frac{\epsilon M}{z}\right] \Omega\left[\frac{-\epsilon z X}{q(1-t)}\right]\right|_{z^{-a}}
$$


Using this we can show that

$$
\mathbf{C}_{a}^{*} h_{n}^{*}= \begin{cases}0 & \text { if } a>1, \\ h_{n-1}^{*} & \text { if } a=1,\end{cases}
$$

and thus (4.16) is none other than

$$
\left\langle E_{n k}, e_{n}\right\rangle=\left\langle E_{n-1, k-1}, h_{n-1}^{*}\right\rangle_{*}=\left\langle E_{n-1, k-1}, e_{n-1}\right\rangle
$$

and an obvious induction argument proves (4.11).

\section{The combinatorial side}

Recall that each Parking functions in $\mathcal{P} \mathcal{F}_{n}$ is obtained by choosing a Dyck path in the $n \times n$ lattice square, then filling the cells adjacent to the NORTH steps of the path with circles (as shown below on the left) and finally filling the circles with the numbers $1,2, \ldots, n$ in a column increasing way (as shown below on the right).
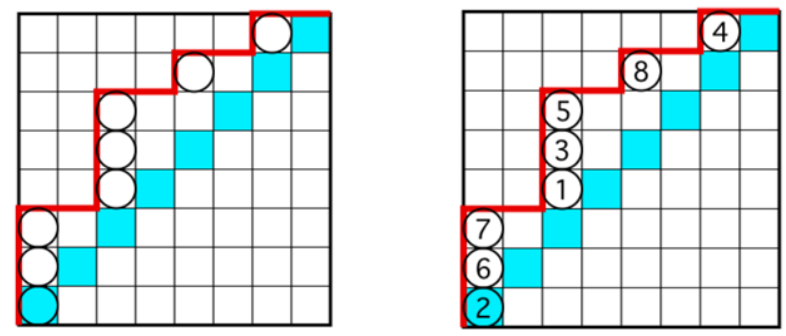

Denote by $\mathcal{P} \mathcal{F}(j, n, k)$ the collection of parking functions whose diagonal permutation $\sigma(P F)$ is a shuffle of $12 \cdots j$ with $(j+1) \cdots(n+j)$ where $n+j$ is in the bottom left corner and there are exactly $k$ of the elements $(j+1) \cdots(n+j)$ in the main diagonal. It will be convenient to refer to $(j+1) \cdots(n+j)$ as the "big" cars and $12 \cdots j$ as the "small" cars. Note that the supporting Dyck path of a parking function $P F \in \mathcal{P F}(j, n, k)$ must have columns of length at most 2 . The reason for this is that, since the big cars are increasing from higher to lower diagonals, none of the big cars can be placed on top of another big car without a violation of the column increasing condition. The same is true for the small cars. Thus we can at most place one of the big cars on top of one of the small cars. This precludes columns of length greater than 2. Recall that there are two ways for two cars to form diagonal inversions: 
1. Two cars are in the same diagonal and the one on the left is smaller than the one on the right. We call these "Primary diagonal inversions."

2. The car on the right is in the immediately lower diagonal and it is smaller than the car on the left. We call these "Secondary diagonal inversions."

Since in a parking function $P F \in \mathcal{P} \mathcal{F}(j, n, k)$ the small cars as well as the big cars increase from right to left along diagonals and from higher to lower diagonals, no primary or secondary diagonal inversions can be produced by a pair of small cars nor by a pair of big cars. Thus a primary diagonal inversion can only be obtained by a small car to the left of a big car in the same diagonal and a secondary diagonal inversion can only be obtained by a big car to the left of a small car in the immediately lower diagonal.

In summary, since the only thing that matters is whether the cars are small or big, we may depict these parking functions by replacing all the big cars by a 2 and all the small cars by a 1 as shown below.
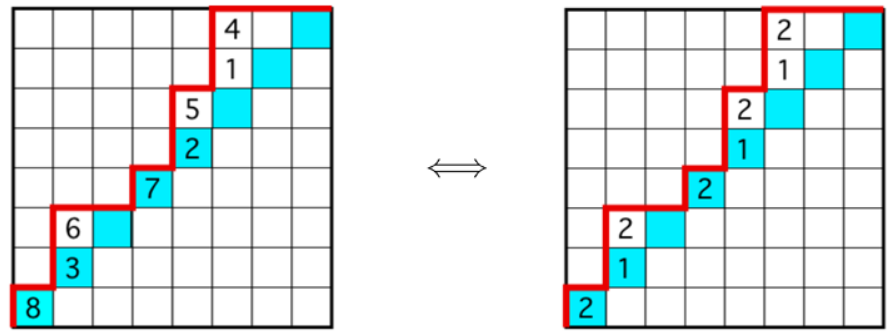

For convenience we will call the latter a "1,2-Parking function" and denote their collection by " $\overline{\mathcal{P} F}(j, n, k)$ ".

It is easy to see that the family $\overline{\mathcal{P F}}(j, n, k)$ is characterized by the following properties

a) There are $j 1$ 's and $n-j 2$ 's.

b) $k$ of the 2's are in the main diagonal, with one of them in the lower left corner.

c) Columns are strictly increasing.

It is also quite evident that, given an element $\overline{P F} \in \overline{\mathcal{P F}}(j, n, k)$, by replacing the 1 's with $1,2, \ldots, j$ and the 2 's by $j+1, j+2, \ldots n$ from higher to lower diagonals and from right to left within diagonals we can reconstruct the element $P F \in \mathcal{P F}(j, n, k)$ that originated it. This allows us to work with $\overline{\mathcal{P} \mathcal{F}}(j, n, k)$ and $P F(j, n, k)$ interchangeably, with the understanding that $\operatorname{dinv}(\overline{P F})=\operatorname{dinv}(P F)$ and area $(\overline{P F})=\operatorname{area}(P F)$. It should also 
be clear than we need not reconstruct $P F$ to obtain area $(\overline{P F})$ since this statistic is given the Dyck path supporting $\overline{P F}$ as well as $P F$. Likewise, our observations concerning diagonal inversions in $\mathcal{P} \mathcal{F}(j, n, k)$ show that $\operatorname{dinv}(\overline{P F})=\operatorname{dinv}(P F)$ can also be computed directly from $\overline{P F}$ itself, by simply counting the pairs $(1,2)$ which form a diagonal inversion.

Keeping all this in mind, let us set

$$
M_{j, n, k}=\sum_{P F \in \mathcal{P F}(j, n, k)} t^{\operatorname{area}(P F)} q^{\operatorname{dinv}(P F)}=\sum_{\overline{P F} \in \overline{\mathcal{P F}}(j, n, k)} t^{\operatorname{area}(\overline{P F})} q^{\operatorname{dinv}(\overline{P F})} .
$$

We are to show that

$$
M_{j, n, k}=H_{j, n, k} .
$$

Given the results of the previous section we need only verify that we have

$$
M_{j, n, k}=t^{n-k} \sum_{s=1}^{j}\left[\begin{array}{c}
k+s-1 \\
s
\end{array}\right]_{q} M_{n-k, j, s}
$$

and

$$
M_{0, n, k}= \begin{cases}0 & \text { if } 1 \leq k<n \\ 1 & \text { if } k=n\end{cases}
$$

Now the latter is easily verified by the following reasoning. Since a $\overline{P F} \in$ $\overline{\mathcal{P F}}(0, n, k)$ has no 1 's, and by property $c)$ we can't have a 2 on top of a 2 , all the 2's must rest in the main diagonal. Thus the collection $\overline{P F}(j, n, k)$ is empty unless $k=n$, in which case its only element is the parking function with all the 2's in the main diagonal. The dinv and area of this parking function are both 0 , and thus (5.4) must hold true precisely as asserted.

We are left to show (5.3). We will do this by constructing a bijection between $\overline{\mathcal{P F}}_{j, n, k}$ and the family $U_{j, n, k}$ consisting of all pairs

$$
U_{j, n, k}=\left\{(w, \overline{P F}): w \in W_{k-1, s} \& \overline{P F} \in \overline{\mathcal{P F}}_{n-k, j, s} \text { with } 1 \leq s \leq j\right\}
$$

where $W_{k-1, s}$ denotes the collection of all 1,2-words with $k-12$ 's and $s$ 1 's.

Our plan is to start with a pair $(w, \overline{P F}) \in U_{j, n, k}$ and construct from it a 1,2-parking function $\Phi(w, \overline{P F}) \in \overline{\mathcal{P F}}_{j, n, k}$ with

$$
\operatorname{weight}(\Phi(w, \overline{P F}))=t^{n-k} \times q^{\operatorname{coinv}(w)} \times t^{\operatorname{area}(\overline{P F})} q^{\operatorname{dinv}(\overline{P F})}
$$


where for $w=w_{1} w_{2} \cdots w_{n}$ we set

$$
\operatorname{coinv}(w)=\sum_{1 \leq i<j \leq n} \chi\left(w_{i}<w_{j}\right)
$$

Since we clearly have the identity

$$
\sum_{(w, \overline{P F}) \in U_{j, n, k}} \operatorname{weight}(w, \overline{P F})=t^{n-k} \sum_{s=1}^{j}\left[\begin{array}{c}
k+s-1 \\
s
\end{array}\right]_{q} M_{n-k, j, s}
$$

to show that $\Phi$ gives our desired bijection we only need to

1. prove that $\Phi$ is onto.

2 . verify that every step of the construction of $\Phi$ is reversible.

3. prove the identity in (5.6).

Our map $\Phi$, is obtained by a procedure which, given a pair $\left(w, \Pi_{1}\right)$ with $w \in W_{k-1, s}$ and $\Pi_{1} \in \overline{\mathcal{P F}}(n-k, j, s)$ constructs the corresponding element $\Phi\left(w, \Pi_{1}\right) \in \overline{\mathcal{P F}}(j, n, k)$ by the following 4 successive steps. For clarity we will illustrate our construction by applying it to the pair $\left(w, \Pi_{1}\right) \in U_{4,5,2}$, when $w$ is any one of the 6 words $2211,2121,2112,1221,1212,1122$ in $W_{2,2}$ and $\Pi_{1}$ is the following element of $\overline{\mathcal{P F}}_{4,5,2}$,

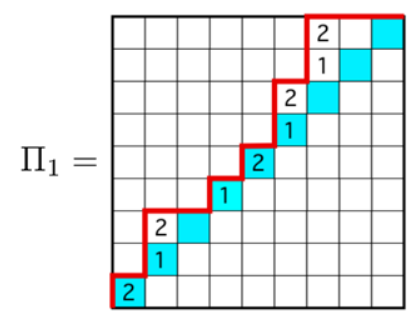

at the end of our construction we will display the corresponding 6 elements of $\overline{\mathcal{P F}}_{5,7,3}$.

Step 1 Move all the 1's one cell to the left and modify the Dyck path so that the 1's are adjacent to its NORTH steps. Call the resulting lattice diagram $\Pi_{2}$. 


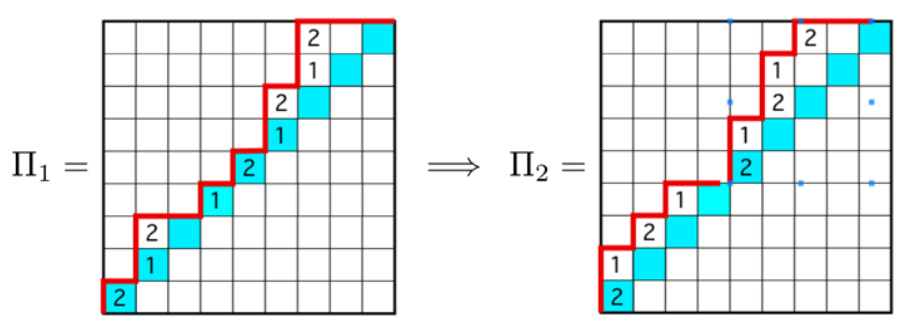

Step 2 Interchange the 1's and the 2's. Call the resulting lattice diagram $\Pi_{3}$. Note that since all the ones have moved and $\Pi_{1}$ had $s$ 's on the main diagonal, the main diagonal of $\Pi_{3}$ will contain $s$ 1's and no 2's.

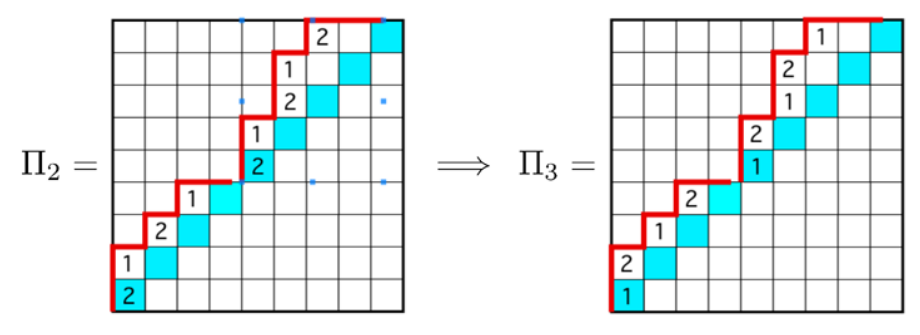

Step 3 Break up the lattice diagram of $\Pi_{3}$ into the $s$ sub-diagram, which start at the 1's in the main diagonal, as shown below.

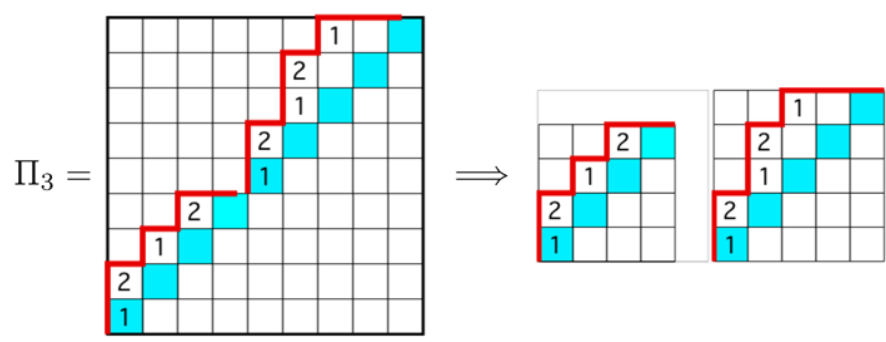

Step 4 Given a word $w \in W_{k-1, s}$ by moving the $s$ sub-diagrams NORHEAST insert $k-12$ 's in the main diagonal in between the sub-diagrams in exactly the same way the 2's are in between the 1's in $w$. This done prepend a 2 on the bottom left of the resulting lattice diagrams.

In the following display we depict the results of applying Step 4 with each of the six words in $W_{2,2}$. Here the lattice cell containing the prepended last 2 is thickened. 

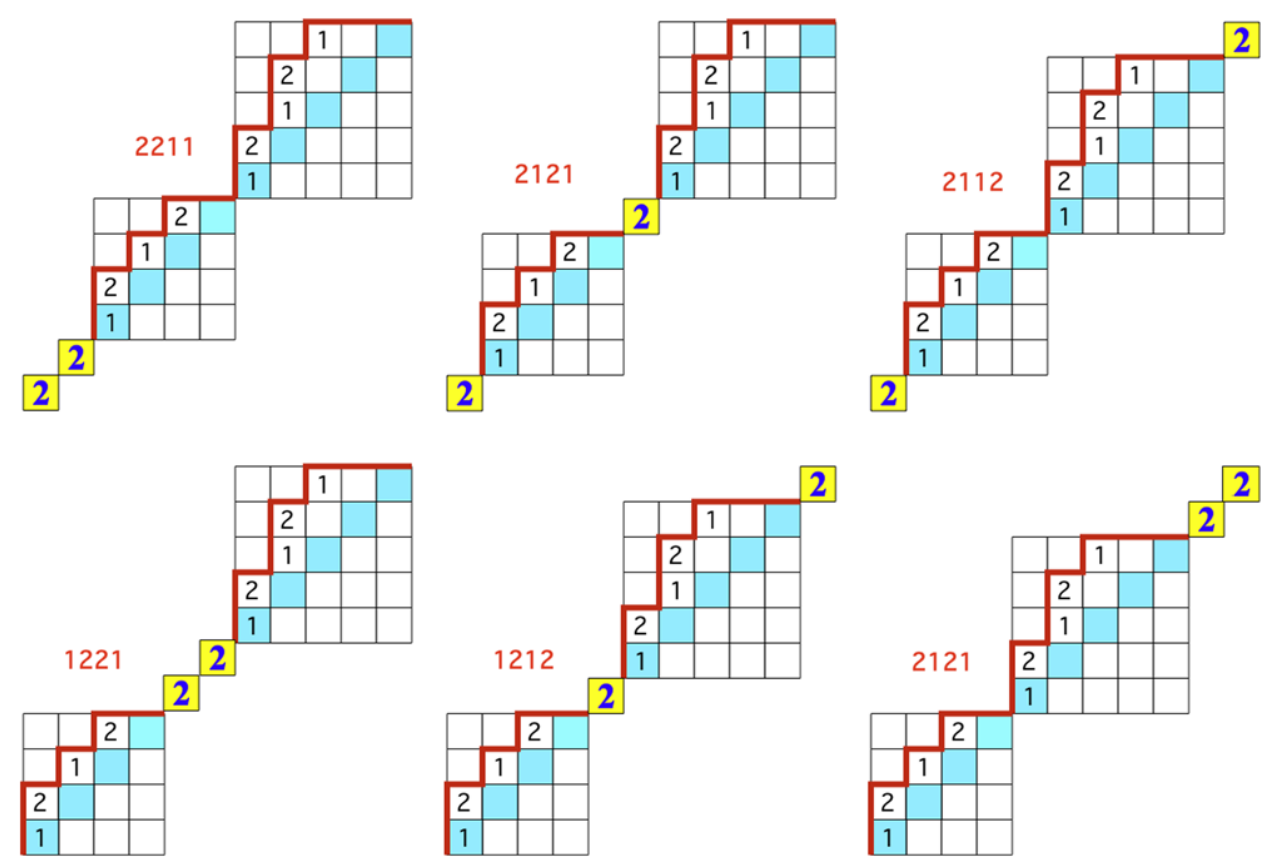

1) $\Phi$ is onto

We need only to show that the increasing column condition is satisfied after the second step of our procedure. Since we move only the 1's, the columns in $\Pi_{2}$ consist of 2 's in the same column as they were in $\Pi_{1}$ and the 1's which occured one column to the right in $\Pi_{1}$. Since any column in $\Pi_{1}$ has at most a single 1 and a single 2 , the same must be true of $\Pi_{2}$. In any column in $\Pi_{2}$ containing one of each, the 1 must be on top of the 2 (or else the path corresponding to $\Pi_{1}$ had a west step). This gives us that $\Pi_{3}$ satisfies the increasing column condition.

2) Every step of the construction of $\Phi$ is reversible

Given a 1,2-Parking Function $P F \in \mathcal{P F}(j, n, k)$, the number of ones in the main diagonal yields $s$ and the relative position of the 1's and 2's in the main diagonal determine the corresponding word $w \in W_{k-1, s}$. This given, the removal of the two's will bring us right back to the end of Step 2. Finally, carrying out Step 2 and Step 1 successively and in reverse yields the element $\Pi_{1} \in \overline{\mathcal{P F}}_{n-k, j, s}$ needed in the pair $(w, \overline{P F}) \in U_{j, n, k}$. To see this, note that the first element in the main diagonal of $P F$ that isn't removed must be a 1 . This 1 will become the required 2 in the $(1,1)$ cell of $\overline{P F}$. We have thus shown that $\Phi$ is invertible. 
3) Proof of the identity in (5.6)

We are to show that

$$
\text { weight }(\Phi(w, \overline{P F}))=t^{n-k} \times q^{\operatorname{coinv}(w)} \times t^{\operatorname{area}(\overline{P F})} q^{\operatorname{dinv}(\overline{P F})} .
$$

First, note that the area increases by $n-k$ by from $\Pi_{1}$ to $\Pi_{2}$, in Step 1 , due the westerly motion of the $n-k 1^{\prime} s$. Since all the elements added afterwards are inserted in the main diagonal there are no further area changes and the increase in area from $\overline{P F}$ to $P F$ is precisely $n-k$. This accounts for the factor $t^{n-k}$. Note further that there is no change from $\Pi_{1}$ to $\Pi_{3}$ since the combination of Step 1 and Step 2 replaces primary diagonal inversions with secondary ones and vice versa as seen in the diagrams below.

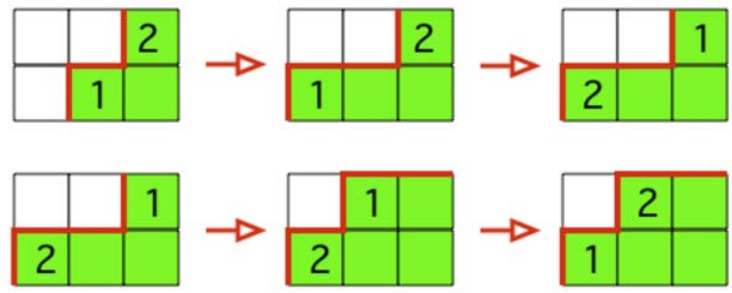

However in Step 4 the insertion of the $k-12$ 's adds coinv $(w)$ to the change due to the interaction of these 2's with the 1's in the main diagonal. This accounts for the factor $q^{\operatorname{coinv}(w)}$ in (5.6).

This completes our proof of the combinatorial recursion in (5.3).

\section{References}

[1] Bergeron, F. and Garsia, A. M. (1999). Science fiction and Macdonald's polynomials, Algebraic methods and q-special functions (Montréal, QC, 1996), CRM Proc. Lecture Notes, vol. 22, Amer. Math. Soc., Providence, RI, pp. 1-52. MR1726826

[2] Bergeron, F., Garsia, A. M., Haiman, M., and Tesler, G. (1999). Identities and positivity conjectures for some remarkable operators in the theory of symmetric functions. Methods in Appl. Anal. 6 363-420. MR1803316

[3] Garsia, A. M. and Haglund, J. (2002). A proof of the q,t-Catalan positivity conjecture. Discrete Math. 256 677-717. MR1935784 
[4] Garsia, A. M. and Haiman, M. (1996). Some Natural Bigraded $S_{n^{-}}$ Modules and q,t-Kostka Coefficients. (electronic) The Foata Festschrift, http://www. combinatorics.org/Volume_3/PDF/v3i2r24.pdf.

[5] Garsia, A. M. and Haiman, M. (1996). A remarkable q,t-Catalan sequence and q-Lagrange inversion. J. Algebraic Combin. 5(3) 191-244. MR1394305

[6] Garsia, A., Haiman, M., and Tesler, G. (1999). Explicit Plethystic Formulas for the Macdonald q,t-Kostka Coefficients, Séminaire Lotharingien de Combinatoire, B42m, 45 pp. MR1701592

[7] Garsia, A. M. and Tesler, G. (1996). Plethystic formulas for Macdonald q,t-Kostka coefficients, Adv. Math. 123, 144-222. MR1420484

[8] Garsia, A. M., Xin, G., and Zabrocki, M. (2011). Hall-Littlewood operators in the theory of parking functions and diagonal harmonics, Int. Math. Res. Notices, doi:10.1093/imrn/rnr060.

[9] Gessel, I. (1984). Multipartite P-partitions and inner products of skew Schur functions, in: C. Greene (Ed.), Combinatorics and Algebra, in: Contemp. Math., vol. 34, pp. 289-301. MR0777705

[10] Haglund, J. (2004). A proof of the q,t-Schröder conjecture. Internat. Math. Res. Notices 11 525-560. MR2038776

[11] Haglund, J. (2008). The q,t-Catalan Numbers and the Space of Diagonal Harmonics, AMS University Lecture Series, vol. 41, pp. 167. MR2371044

[12] Haglund, J., Haiman, M., Loehr, N., Remmel, J. B., and Ulyanov, A. (2005). A combinatorial formula for the character of the diagonal coinvariants. Duke J. Math. 126 195-232. MR2115257

[13] Haiman, M. (2001). Hilbert schemes, polygraphs, and the Macdonald positivity conjecture. J. Amer. Math. Soc. 14 941-1006. MR1839919

[14] Haglund, J., Morse, J., and Zabrocki, M. A compositional refinement of the Shuffle Conjecture, The Canadian Journal of Math., doi:10.4153/CJM-2011-078-4, pp. 23.

[15] Macdonald, I. G. (1995). Symmetric functions and Hall polynomials. In: Oxford Mathematical Monographs, 2nd ed. Oxford Science Publications, The Clarendon Press Oxford University Press, New York. MR1354144 


\author{
Adriano Garsia \\ Mathematics Department \\ University of California, San Diego \\ 9500 Gilman Drive \# 0112 \\ LA Jolla, CA 92093-0112 \\ USA \\ E-mail address: garsia@math.ucsd.edu \\ Angela Hicks \\ Mathematics Department \\ University of California, San Diego \\ USA \\ E-mail address: ashicks@math.ucsd.edu \\ AndRew STOUT \\ Mathematics Department \\ University of California, San Diego \\ USA \\ E-mail address: astout@math.ucsd.edu \\ Received September 9, 2010
}

